This item was submitted to Loughborough's Research Repository by the author.

Items in Figshare are protected by copyright, with all rights reserved, unless otherwise indicated.

\title{
Normal forms for wave motion in fluid interfaces
}

PLEASE CITE THE PUBLISHED VERSION

LICENCE

CC BY-NC-ND 4.0

\section{REPOSITORY RECORD}

Craig, Walter, and Mark D. Groves. 2019. "Normal Forms for Wave Motion in Fluid Interfaces". figshare. https://hdl.handle.net/2134/804. 


\title{
Loughborough $\checkmark$ University
}

\section{Normal forms for wave motion in fluid interfaces}

\author{
Walter Craig ${ }^{1,2}$ \\ Mathematics Department and Lefschetz Center for Dynamical Systems, \\ Brown University, Providence RI 02912, USA \\ Mark D. Groves ${ }^{2}$ \\ Department of Mathematical Sciences, Loughborough University, \\ Loughborough LE11 3TU, UK
}

\begin{abstract}
The subject of this paper is the dynamics of wave motion in the two-dimensional Kelvin-Helmholtz problem for an interface between two immiscible fluids of different densities. The difference of the mean flow between the two fluid bodies is taken to be zero, and the effects of surface tension are neglected. We transform the problem to Birkhoff normal form, in which a precise analysis can be made of classes of resonant solutions. This paper studies standing-wave solutions of the fourth-order normal form in particular detail. We find that that there are families of invariant resonant subsystems, which are nevertheless integrable. Within these families we describe the periodic and the time quasi-periodic standing waves, and determine their stability or instability. In particular we show that for a certain range of densities, a basic time-periodic standing wave with principal wave number $k$ is unstable to modes with principal wave numbers $k / 4$ and $9 k / 4$, and we calculate the Lyapunov exponent of the instability. We furthermore show that the stable and unstable manifolds to these periodic solutions of the Birkhoff normal form are connected by a homoclinic orbit. This instability mechanism, as well as others that we describe, appears to be new, and its description is possible because of the precision afforded by the normal form. These results contrast with the case of the water wave problem described by Dyachenko \& Zakharov [1] and Craig \& Worfolk [2], where the fourth-order Birkhoff normal form is an integrable system, with all orbits undergoing stable almost-periodic motion, and instabilities arise only in normal forms to higher order.
\end{abstract}

$\overline{1}$ Research supported in part by the NSF under grant DMS 9501514.

2 Research supported in part by the EPSRC under grant GR/K98254. 


\section{Introduction}

This paper is a study of the dynamics of wave motion in the two-dimensional Kelvin-Helmholtz problem for the evolution of an interface between two immiscible fluids of different densities; the effects of surface tension are neglected. We consider motions of the interface which are periodic in the spatial variable, with fixed period. When Neumann boundary conditions are imposed on two vertical confining walls this is the problem of standing waves, and when periodic boundary conditions are imposed, the problem admits general spatially periodic solutions, including the travelling waves. The theory of normal forms in this paper applies to both of these settings, however we focus our subsequent analysis for the most part on standing waves in the fluid interface.

Previous work on standing waves in fluids includes the systematic approach of Tadjbakhsh \& Keller [3], who gave a description of the simplest one-mode standing wave in a free surface. This description was extended to the Kelvin Helmholtz problem of a fluid interface by Thorpe [4], and by Drazin [5]; the former article also includes experimental descriptions and photographs of onemode standing interfacial waves in wavetanks. Their work is in the regime of small density differences between the fluids. Miles [6] sets the Kelvin Helmholtz problem in a Lagrangian framework, recovering Thorpe's expression for the amplitude/frequency dependence of one-mode solutions. In more recent work, Dias \& Bridges [7] examined standing and travelling waves of the Kelvin Helmholtz problem in a Hamiltonian setting, and discussed approximations to free interface motions which involve a low number of spatial modes. The present paper examines the class of standing wave solutions in much greater detail, admitting arbitrary density differences and having no a priori truncation of spatial modes. It also provides a precise statement of the stability of solutions.

The starting point of our analysis is the description by Benjamin \& Bridges [8] of the equations of motion as a Hamiltonian system. This is closely related to the original description given by Zakharov [9] for the water-wave problem. The analysis of small-amplitude solutions is evocative of the theory of Hamiltonian systems in the neighborhood of an elliptic stationary point; note, however, that in the case at hand there are infinitely many degrees of freedom. It is therefore natural to apply transformation theory to the problem to eliminate all nonessential nonlinearities and to study the evolution described by the resulting Birkhoff normal forms. This point of view was adopted by Craig \& Worfolk [2] and Craig [10] for the water-wave problem.

As in the water-wave problem, there are no cubic resonant terms (or threewave interactions) for the Kelvin-Helmholtz problem, and all cubic nonlinear terms in the Hamiltonian may therefore be eliminated by an appropriate choice 
of canonical transformation. There are, however, nontrivial quartic resonant terms, which are reflected in the fourth-order Birkhoff normal-form Hamiltonian. In the case of water waves in a deep fluid, there is a remarkable cancellation of nongeneric resonant coefficients at this level (see Dyachenko \& Zakharov [1] and Craig \& Worfolk [2]), and the fourth-order normal form is completely integrable. The first result of the present paper is that this cancellation does not occur for the Kelvin-Helmholtz problem, and the resulting fourth-order Birkhoff normal form is a nonlinear system which possesses nontrivial resonance terms. Nevertheless there turn out to be infinitely many completely integrable subsystems in resonance, at least in the case of the restriction to standing waves. These subsystems possess Poisson commuting integrals which are relatively unrelated to the action-angle variables of the linearised problem.

We analyse the structure of the above finite-dimensional invariant subsystems of the fourth-order normal form in detail. They are either simple or have the resonance structure $\left(a:(a+1): a(a+1): a^{2}+a+1\right)$ for some $a \in \mathbb{N}$. For $a=1$ this resonance involves three degrees of freedom and the $(1: 2: 3)$ resonance, while for $a>1$ it represents a four-degree-of-freedom system; all of these subsystems possess complete sets of integrals of motion. Most solutions to a subsystem are quasi-periodic in time; however, depending on the dimensionless parameter $r=\left(\rho^{2}+\rho_{1}^{2}\right) / \rho \rho_{1}$, where $\rho$ and $\rho_{1}$ are respectively the densities of the lower and upper fluids, the integrals may degenerate at certain points in phase space. There are therefore lower dimensional tori, on which there is periodic motion or quasi-periodic motion with fewer frequencies, and some of these singular tori are unstable, being 'whiskered' by homoclinic orbits with positive Lyapunov exponent. In particular, for $63 / 16-3 \sqrt{3} / 4<r<63 / 16+3 \sqrt{3} / 4$ the basic time periodic standing wave solution with principal wave number $4 k$ is unstable to modes with principal spatial wave numbers $k$ and $9 k$. The stable and unstable manifolds to this unstable periodic orbit are computed explicitly together with the Lyapunov exponent of the instability. Furthermore, these stable and unstable manifolds are connected by a homoclinic orbit lying in the resonant subsystem. A number of similar phenomena of resonance and instability are also described in the more general four-degree-of-freedom resonant subsystems. This phenomenon of instability and homoclinic orbits again offers a contrast with the case of the water-wave problem, in which the fourth-order normal form has the property that all solutions are almost periodic.

The Birkhoff normal forms for the Kelvin-Helmholtz problem are approximations of the Euler equations with a free interface, and their solutions are intended only as approximations of Euler flow. Nevertheless, many features of the flow should be accurately represented by solutions of the systems given by the normal forms. In particular, the instabilities of standing waves mentioned above can be expected to persist, as can the nondegeneracy of the nonlinear relationship between the amplitude of solutions and their frequencies (the 
Arnold condition) for the resonant subsystems. Among the many methods of approximating the evolution of free surfaces, our approach is distinguished by the property that it is uniform in wave number. Moreover, all canonical transformations are exact and genuinely infinite dimensional, so that in principle no information is lost. This contrasts with the work of Dias \& Bridges [7], who also use a Hamiltonian formulation to study standing and travelling waves in fluid interfaces, but their analysis is based upon a truncation to a finite number of Fourier modes. Our approximations to Euler flow are within the weakly nonlinear regime, and are effectively the approximation of a Hamiltonian by Taylor polynomials about a stationary point.

\section{The hydrodynamic problem and its Hamiltonian formulation}

The problem concerns two infinite, immiscible, perfect fluids separated by an interface $\{y=\eta(x)\}$; the fluid motion is two-dimensional and the densities of the upper and lower fluids are respectively $\rho_{1}$ and $\rho$ with $\rho_{1}<\rho$. We assume that the interface is spatially periodic, so that $\eta(x+2 \pi, t)=\eta(x, t)$ and the fluid domains are $S_{1}(\eta)=\{0 \leq x<2 \pi, \eta(x)<y<+\infty\}$ and $S(\eta)=\{0 \leq x<2 \pi,-\infty<y<\eta(x)\}$. Within each fluid domain the evolution is given by potential flow, so that

$$
\begin{array}{llll}
\vec{u}=\nabla \varphi_{1}, & \Delta \varphi_{1}=0 & \text { within } & S_{1}(\eta), \\
\vec{u}=\nabla \varphi, & \Delta \varphi=0 & \text { within } & S(\eta),
\end{array}
$$

where $\varphi_{1}(x+2 \pi, y, t)=\varphi_{1}(x, y, t), \varphi(x+2 \pi, y, t)=\varphi(x, y, t)$. The fluid interface obeys the kinematic equations

$$
\begin{aligned}
& \partial_{t} \eta=-\partial_{y} \varphi_{1}+\partial_{x} \eta \partial_{x} \varphi_{1}, \\
& \partial_{t} \eta=\partial_{y} \varphi-\partial_{x} \eta \partial_{x} \varphi
\end{aligned}
$$

and the Bernoulli condition that

$$
\rho_{1}\left(\partial_{t} \varphi_{1}+\frac{1}{2}\left(\nabla \varphi_{1}\right)^{2}+g \eta\right)=\rho\left(\partial_{t} \varphi+\frac{1}{2}(\nabla \varphi)^{2}+g \eta\right) .
$$

We consider flows for which the mean velocity vanishes; in particular the mean velocity shear between the two fluids is zero and $\vec{u} \rightarrow 0$ as $y \rightarrow \pm \infty$. The special case of standing waves is treated in detail; here one imposes Neumann boundary conditions on confining vertical walls, so that

$$
\begin{aligned}
-\partial_{x} \varphi_{1}(0, y, t) & =0=\partial_{x} \varphi_{1}(\pi, y, t) \\
-\partial_{x} \varphi(0, y, t) & =0=\partial_{x} \varphi(\pi, y, t) .
\end{aligned}
$$


The kinetic energy of the system in each fluid domain is given by the Dirichlet integrals

$$
K_{1}=\frac{1}{2} \int_{S_{1}(\eta)} \rho_{1}\left|\nabla \varphi_{1}(x, y)\right|^{2} \mathrm{~d} y \mathrm{~d} x, \quad K=\frac{1}{2} \int_{S(\eta)} \rho|\nabla \varphi(x, y)|^{2} \mathrm{~d} y \mathrm{~d} x
$$

and the potential energy of the system is

$$
V=\frac{1}{2} \int_{0}^{2 \pi} g\left(\rho-\rho_{1}\right) \eta^{2} \mathrm{~d} x
$$

The Hamiltonian function is the total energy

$$
H=K_{1}+K+V
$$

and the only real subtlety is the choice of canonical conjugate variables. Denote the boundary values of the velocity potentials by $\Phi_{1}(x)=\varphi_{1}(x, \eta(x))$ and $\Phi(x)=\varphi(x, \eta(x))$. Following Benjamin \& Bridges [8] we set

$$
\xi(x)=\rho \Phi(x)-\rho_{1} \Phi_{1}(x),
$$

and the natural choice of canonical variables is $(\eta, \xi)$. To express the kinetic energy in an explicit form in terms of the canonical variables, let us introduce Dirichlet-Neumann operators $G(\eta)$ and $G^{(1)}(\eta)$ for the two fluid domains $S(\eta)$ and $S_{1}(\eta)$ (cf. Craig \& Sulem [11]). Given boundary data $\Phi(x)$ on the surface $\{y=\eta(x)\}$ of the domain $S(\eta)$, let $\varphi(x, y)$ be the harmonic function extending $\Phi(x)$ to $S(\eta)$ which is $2 \pi$-periodic in $x$ and decays to zero as $y \rightarrow-\infty$. The Dirichlet-Neumann operator for the domain $S(\eta)$ returns the exterior normal derivative of the potential function $\varphi$ from its boundary data $\Phi(x)$, so that

$$
G(\eta) \Phi(x)=\left(1+\left(\partial_{x} \eta\right)^{2}\right)^{\frac{1}{2}}(\nabla \varphi \cdot \vec{N})(x)
$$

There is a similar definition for the domain $S_{1}(\eta)$ in terms of the exterior normal derivative of $\varphi_{1}(x, y)$, namely

$$
G^{(1)}(\eta) \Phi_{1}(x)=\left(1+\left(\partial_{x} \eta\right)^{2}\right)^{\frac{1}{2}}\left(\nabla \varphi_{1} \cdot \vec{N}_{1}\right)(x) .
$$

Since $N_{1}=-N$ it follows from equations (1), (2), (5) and (6) that

$$
G(\eta) \Phi(x)+G^{(1)}(\eta) \Phi_{1}(x)=0 .
$$


Using the definition (4) of $\xi(x)$ and the identity (7), we find that

$$
\begin{aligned}
G(\eta) \xi(x) & =\rho G(\eta) \Phi(x)-\rho_{1} G(\eta) \Phi_{1}(x) \\
& =-\left(\rho G^{(1)}(\eta)+\rho_{1} G(\eta)\right) \Phi_{1}(x),
\end{aligned}
$$

so that

$$
\Phi_{1}(x)=-B^{-1} G(\eta) \xi(x),
$$

where $B(\eta):=\rho G^{(1)}(\eta)+\rho_{1} G(\eta)$. Similarly, one finds that

$$
\Phi(x)=B^{-1} G^{(1)}(\eta) \xi .
$$

The Dirichlet integrals for the two fluid domains are conveniently described in terms of the operators $G(\eta)$ and $G^{(1)}(\eta)$ by the formulae

$$
K=\frac{1}{2} \int_{0}^{2 \pi} \rho \Phi(x) G(\eta) \Phi(x) \mathrm{d} x, \quad K_{1}=\frac{1}{2} \int_{0}^{2 \pi} \rho_{1} \Phi_{1}(x) G^{(1)}(\eta) \Phi_{1}(x) \mathrm{d} x
$$

(see Craig \& Sulem [11]). It follows from equation (3) that

$$
\begin{aligned}
& H(\eta, \xi)=\frac{1}{2} \int_{0}^{2 \pi} \rho \Phi(x) G(\eta) \Phi(x) \mathrm{d} x \\
& +\frac{1}{2} \int_{0}^{2 \pi} \rho_{1} \Phi_{1}(x) G^{(1)}(\eta) \Phi_{1}(x) \mathrm{d} x+\frac{1}{2} \int_{0}^{2 \pi} g\left(\rho-\rho_{1}\right) \eta^{2} \mathrm{~d} x,
\end{aligned}
$$

and the following result is verified by substituting the formulae (8), (9) into this equation.

Theorem 1 The Kelvin-Helmholtz problem can be described as a Hamiltonian system with Hamiltonian

$$
H(\eta, \xi)=\frac{1}{2} \int_{0}^{2 \pi} \xi(x) G(\eta) B^{-1} G^{(1)}(\eta) \xi(x) \mathrm{d} x+\frac{1}{2} \int_{0}^{2 \pi} g\left(\rho-\rho_{1}\right) \eta^{2} \mathrm{~d} x .
$$

The evolution equations for the free interface are equivalent to Hamilton's canonical equations

$$
\partial_{t} \eta=\delta_{\xi} H, \quad \partial_{t} \xi=-\delta_{\eta} H
$$


As in the papers by Craig \& Groves [12] and Craig \& Worfolk [2], our analysis involves the expansion of the Hamiltonian $H(\eta, \xi)$ in a Taylor series in the variables $(\eta, \xi)$ about zero. The potential energy is clearly quadratic in $\eta$, and the effort lies in understanding the Taylor expansion of the operator $G(\eta) B^{-1} G^{(1)}(\eta)$ appearing in the expression for the total kinetic energy. As discussed in Craig \& Sulem [11], the operators $G(\eta)$ and $G^{(1)}(\eta)$ depend analytically on the variable $\eta \in \operatorname{Lip}(0,2 \pi)$, and therefore

$$
G(\eta)=\sum_{j \geq 0} G_{j}(\eta)
$$

where the Taylor polynomials $G_{j}(\eta)$ are homogeneous of degree $j$ in $\eta$. The relation

$$
G^{(1)}(\eta)=G(-\eta)
$$

follows from the fact that the geometries of $S_{1}(\eta)$ and $S(-\eta)$ are identical, and one deduces that

$$
G^{(1)}(\eta)=\sum_{j \geq 0}(-1)^{j} G_{j}(\eta) .
$$

Using the definition of $B(\eta)$ and (10), (11), we find that

$$
\begin{aligned}
B^{-1}(\eta)= & \frac{1}{\rho+\rho_{1}} G_{0}^{-1}+\frac{\rho-\rho_{1}}{\left(\rho+\rho_{1}\right)^{2}} G_{0}^{-1} G_{1}(\eta) G_{0}^{-1} \\
& -\frac{1}{\rho+\rho_{1}} G_{0}^{-1} G_{2}(\eta) G_{0}^{-1}+\frac{\left(\rho-\rho_{1}\right)^{2}}{\left(\rho+\rho_{1}\right)^{3}} G_{0}^{-1} G_{1}(\eta) G_{0}^{-1} G_{1}(\eta) G_{0}^{-1} \\
& +O\left(|\eta|^{3}\right),
\end{aligned}
$$

from which it follows that

$$
\begin{aligned}
G(\eta) B^{-1}(\eta) G^{(1)}(\eta)= & \frac{1}{\rho+\rho_{1}} G_{0}+\frac{\rho-\rho_{1}}{\left(\rho+\rho_{1}\right)^{2}} G_{1}(\eta) \\
& +\left(\frac{1}{\rho+\rho_{1}} G_{2}(\eta)-\frac{4 \rho \rho_{1}}{\left(\rho-\rho_{1}\right)^{3}} G_{1}(\eta) G_{0}^{-1} G_{1}(\eta)\right) \\
& +O\left(|\eta|^{3}\right) .
\end{aligned}
$$

The next result is an immediate consequence of (12).

Theorem 2 The Taylor expansion of the Hamiltonian is given up to quartic terms by the expression

$$
H=H_{2}(\eta, \xi)+H_{3}(\eta, \xi)+H_{4}(\eta, \xi)+R_{5},
$$


where

$$
\begin{aligned}
H_{2}(\eta, \xi)= & \int_{0}^{2 \pi}\left(\frac{1}{2\left(\rho+\rho_{1}\right)} \xi(x) G_{0} \xi(x)+\frac{g}{2}\left(\rho-\rho_{1}\right) \eta^{2}(x)\right) \mathrm{d} x, \\
H_{3}(\eta, \xi)= & \int_{0}^{2 \pi}\left(\frac{\rho-\rho_{1}}{2\left(\rho+\rho_{1}\right)^{2}} \xi(x) G_{1}(\eta) \xi(x)\right) \mathrm{d} x, \\
H_{4}(\eta, \xi)= & \int_{0}^{2 \pi}\left(\frac{1}{2\left(\rho+\rho_{1}\right)} \xi(x) G_{2}(\eta) \xi(x)\right. \\
& \left.-\frac{4 \rho \rho_{1}}{2\left(\rho+\rho_{1}\right)^{3}} \xi(x) G_{1}(\eta) G_{0}^{-1} G_{1}(\eta) \xi(x)\right) \mathrm{d} x,
\end{aligned}
$$

and $R_{5}$ is the Taylor remainder term.

When $\eta$ is smooth, explicit expressions for the terms in the Taylor expansion of $G(\eta)$ can be computed using recursion formulae derived by Craig \& Sulem [11]. The relevant formulae are

$$
\begin{aligned}
G_{0}(D) & =|D|, \\
G_{1}(\eta) & =D \eta(x) D-G_{0}(D) \eta(x) G_{0}(D), \\
G_{2}(\eta) & =-\frac{1}{2}\left(D^{2} \eta^{2}(x) G_{0}(D)+G_{0}(D) \eta^{2}(x) D^{2}\right. \\
& \left.\quad-2 G_{0}(D) \eta(x) G_{0}(D) \eta(x) G_{0}(D)\right),
\end{aligned}
$$

in which $D=-\mathrm{i} \partial_{x}$ and $G_{0}(D)$ represent Fourier-multiplier operators. Using the fact that $D G_{0}^{-1}(D) D=G_{0}(D)$, one finds after a short calculation that the quadratic term on the right-hand side of (12) is

$$
\begin{aligned}
& \frac{1}{\rho+\rho_{1}} G_{2}(\eta)-\frac{4 \rho \rho_{1}}{\left(\rho-\rho_{1}\right)^{3}} G_{1}(\eta) G_{0}^{-1} G_{1}(\eta) \\
= & \frac{1}{2\left(\rho+\rho_{1}\right)}\left(-D^{2} \eta^{2}(x) G_{0}-G_{0} \eta^{2}(x) D^{2}+2 G_{0} \eta(x) G_{0} \eta(x) G_{0}\right) \\
& -\frac{4 \rho \rho_{1}}{\left(\rho+\rho_{1}\right)^{3}}\left(D \eta(x) G_{0} \eta(x) D-D \eta(x) D \eta(x) G_{0}\right. \\
& \left.\quad-G_{0} \eta(x) D \eta(x) D+G_{0} \eta(x) G_{0} \eta(x) G_{0}\right) .
\end{aligned}
$$

The next step is to express the $2 \pi$-periodic functions $(\eta, \xi)$ in Fourier series

$$
\eta(x)=\frac{1}{\sqrt{2 \pi}} \sum_{k \in \mathbb{Z}} \eta(k) \mathrm{e}^{\mathrm{i} k x}, \quad \xi(x)=\frac{1}{\sqrt{2 \pi}} \sum_{k \in \mathbb{Z}} \xi(k) \mathrm{e}^{\mathrm{i} k x} .
$$


The Fourier coefficients satisfy the reality condition

$$
\eta(-k)=\eta^{*}(k), \quad \xi(-k)=\xi^{*}(k),
$$

where $\mathrm{i}^{*}=-\mathrm{i}$ denotes complex conjugation; the quantities $\eta(0)$ and $\xi(0)$ are conserved and without loss of generality we will take $\eta(0)=0$ and $\xi(0)=0$ for the remainder of this paper. Hamilton's equations become

$$
\partial_{t} \eta(k)=\delta_{\xi^{*}(k)} H, \quad \partial_{t} \xi(k)=-\delta_{\eta^{*}(k)} H, \quad k>0 .
$$

Expressed in Fourier series, the quadratic part of the Hamiltonian (14) is

$$
H_{2}(\eta, \xi)=\sum_{k>0}\left(\frac{k}{\left(\rho+\rho_{1}\right)} \xi(k) \xi^{*}(k)+g\left(\rho-\rho_{1}\right) \eta(k) \eta^{*}(k)\right),
$$

and the corresponding linearised equations of motion are

$$
\begin{aligned}
& \partial_{t} \eta(k)=\delta_{\xi^{*}(k)} H_{2}=\frac{k}{\rho+\rho_{1}} \xi(k), \\
& \partial_{t} \xi(k)=-\delta_{\eta^{*}(k)} H_{2}=-g\left(\rho-\rho_{1}\right) \eta(k) .
\end{aligned}
$$

This system is a harmonic oscillator with infinitely many degrees of freedom.

Proposition 3 All solutions of the linearised problem (23), (24) are almost periodic with frequencies

$$
\omega(k)=\sqrt{\frac{g\left(\rho-\rho_{1}\right) k}{\left(\rho+\rho_{1}\right)}}, \quad k>0 .
$$

Introduce a complex symplectic unit $\mathrm{j}$ with conjugate $\overline{\mathrm{j}}=-\mathrm{j}$ and complex symplectic coordinates

$$
\begin{array}{r}
z(k)=\sqrt[4]{\frac{g\left(\rho-\rho_{1}\right)\left(\rho+\rho_{1}\right)}{4|k|}}(\eta(k)+\eta(-k)) \\
+\mathrm{j} \sqrt[4]{\frac{|k|}{4 g\left(\rho-\rho_{1}\right)\left(\rho+\rho_{1}\right)}}(\xi(k)+\xi(-k)), \\
w(k)=\frac{1}{\mathrm{i}} \sqrt[4]{\frac{g\left(\rho-\rho_{1}\right)\left(\rho+\rho_{1}\right)}{4|k|}}(\eta(k)-\eta(-k)) \\
+\frac{\mathrm{j}}{\mathrm{i}} \sqrt[4]{\frac{|k|}{4 g\left(\rho-\rho_{1}\right)\left(\rho+\rho_{1}\right)}}(\xi(k)-\xi(-k)),
\end{array}
$$


and note that

$$
z(k)=z^{*}(k)=z(-k), \quad w(k)=w^{*}(k)=-w(-k) .
$$

In these variables Hamilton's equations are

$$
\partial_{t} z(k)=-2 \mathrm{j} \partial_{\bar{z}(k)} H, \quad \partial_{t} w(k)=-2 \mathrm{j} \partial_{\bar{w}(k)} H, \quad k>0,
$$

and the Poisson bracket between two functionals $P, Q$ is given by

$$
\begin{aligned}
\{P, Q\}=-2 \mathrm{j} \sum_{k>0}\left(\partial_{\bar{z}(k)} P \partial_{z(k)} Q-\partial_{z(k)} P \partial_{\bar{z}(k)} Q\right. \\
\left.+\partial_{\bar{w}(k)} P \partial_{w(k)} Q-\partial_{w(k)} P \partial_{\bar{w}(k)} Q\right) .
\end{aligned}
$$

Substituting (26), (27) into the expressions (14), (15) for $H_{2}$ and $H_{3}$ and using (17), (18), we find that

$$
\begin{aligned}
& H_{2}=\sum_{k>0} \frac{1}{2} \omega(k)(z(k) \bar{z}(k)+w(k) \bar{w}(k)), \\
& H_{3}=\frac{1}{32 \sqrt{\pi}} \sqrt[4]{\frac{g\left(\rho-\rho_{1}\right)^{5}}{\left(\rho+\rho_{1}\right)^{7}}} \sum_{k, m>0}\left(k^{3} m^{3}(k+m)\right)^{\frac{1}{4}} \\
& \quad \times\left(\alpha(k) \alpha(m) \beta^{*}(k+m)+\alpha^{*}(k) \alpha^{*}(m) \beta(k+m)\right),
\end{aligned}
$$

where

$$
\begin{aligned}
& \alpha(k)=z(k)-\bar{z}(k)+\mathrm{i}(w(k)-\bar{w}(k)), \\
& \beta(k)=z(k)+\bar{z}(k)+\mathrm{i}(w(k)+\bar{w}(k)) .
\end{aligned}
$$

We note that $H_{3}=\sqrt[4]{\left(\rho-\rho_{1}\right)^{5} /\left(\rho+\rho_{1}\right)^{7}} H_{3}^{0}$, where $H_{3}^{0}$ is the corresponding cubic term of the water-wave Hamiltonian given by Craig \& Worfolk [2].

Similar calculations show that

$$
H_{4}=\frac{1}{\left(\rho+\rho_{1}\right)} H_{4}^{0}+A_{4}
$$

in which

$$
H_{4}^{0}=\frac{1}{512 \pi} \sum_{k+\ell+m+n=0}|k||n|(|n|+|k|-2|k+\ell|) \sqrt[4]{\frac{|\ell||m|}{|k||n|}} \alpha(k) \beta(\ell) \beta(m) \alpha(n)
$$

is the quartic term in the water-wave Hamiltonian (Craig \& Worfolk [2]) and 


$$
\begin{aligned}
A_{4}=\frac{\rho \rho_{1}}{64 \pi\left(\rho+\rho_{1}\right)^{3}} \sum_{k+\ell+m+n=0}((|k||n| & -k n)|k+\ell|+(|k| n-k|n|)(k+\ell)) \\
& \times \sqrt[4]{\frac{|\ell||m|}{|k||n|}} \alpha(k) \beta(\ell) \beta(m) \alpha(n)
\end{aligned}
$$

represents additional nonlinear quartic interaction terms which are present in the Kelvin-Helmholtz problem.

\section{Birkhoff normal forms}

\subsection{Transformation theory}

Our basic strategy is to perform a sequence of near-identity canonical transformations on the system (28) in order to eliminate all but the essential nonlinearities from the problem. The method dates from the work of Poincaré, Dulac, Birkhoff and their contemporaries; note, however, that the problem at hand has infinitely many degrees of freedom. Although this paper is concerned only with formal aspects of normal-form transformations, some information is available regarding their convergence (see Craig [10]).

In complex coordinates $(Z, \bar{Z})$ a canonical transformation $Z=f\left(Z_{1}, \bar{Z}_{1}\right)$ is locally given by a generating function $S\left(Z, \bar{Z}_{1}\right)$ such that

$$
\bar{Z}=\partial_{Z} S, \quad Z_{1}=\partial_{\bar{Z}_{1}} S .
$$

We will use a sequence of near-identity transformations with generating functions

$$
S\left(Z, \bar{Z}_{1}\right)=\left\langle Z, \bar{Z}_{1}\right\rangle-2 \mathrm{j} S_{J}\left(Z, \bar{Z}_{1}\right), \quad J=3,4, \ldots
$$

in which $S_{J}$ is homogeneous of degree $J$ and satisfies the reality condition that $S_{J}\left(Z, \bar{Z}_{1}\right)=\overline{S_{J}}\left(\bar{Z}, Z_{1}\right)$. For $J=3,4 \ldots$ the transformations $f_{J}$ given by (30), (31) change the Hamiltonian $H=H_{2}+\sum_{\ell \geq 3} H_{\ell}$ into

$$
\begin{aligned}
H^{(1)}\left(Z_{1}, \bar{Z}_{1}\right)= & H_{2}+\left(H_{3}+\left\{S_{3}, H_{2}\right\}\right) \\
& +\left(H_{4}+\left\{S_{3}, H_{3}\right\}+\frac{1}{2}\left\{S_{3},\left\{S_{3}, H_{2}\right\}\right\}+\left\{S_{4}, H_{2}\right\}\right) \\
& +R_{5}^{(1)}
\end{aligned}
$$

where $R_{5}^{(1)}$ is the fifth-order Taylor remainder term. 
The operator $\operatorname{ad}_{H_{2}}(\cdot)=\left\{H_{2}, \cdot\right\}$ is diagonalised in symplectic complex coordinates. Its action on a monomial $Z^{P} \bar{Z}^{Q}$ whose multi-indices $P=\{p(k)\}_{k>0}$, $Q=\{q(k)\}_{k>0}$ satisfy $|P|+|Q|=J$ is described by

$$
\begin{aligned}
\left\{H_{2}, Z^{P} \bar{Z}^{Q}\right\} & =\left(-\mathrm{j} \sum_{k>0} \omega(k)(p(k)-q(k))\right) Z^{P} \bar{Z}^{Q} \\
& =\left(-\mathrm{j} \sqrt{\frac{g\left(\rho-\rho_{1}\right)}{\rho+\rho_{1}}} \sum_{k>0} \sqrt{k}(p(k)-q(k))\right) Z^{P} \bar{Z}^{Q}
\end{aligned}
$$

the monomial is resonant if $\sum_{k>0} \omega(k)(p(k)-q(k))=0$. The linear space $P_{J}$ of $J$ th-order polynomials decomposes into resonant and nonresonant subspaces, so that $P_{J}=\operatorname{ker}\left(\operatorname{ad}_{H_{2}}\right) \oplus \operatorname{im}\left(\operatorname{ad}_{H_{2}}\right)$, and we write $B=B^{\text {res }}+B^{\text {non }}$ for each $B \in P_{J}$, where $B^{\text {res }} \in \operatorname{ker}\left(\operatorname{ad}_{H_{2}}\right), B^{\text {non }} \in \operatorname{im}\left(\operatorname{ad}_{H_{2}}\right)$. The cohomological equation $\left\{S_{J}, H_{2}\right\}+B=B^{\text {res }}$ appearing at each order in (32) is solved by choosing $S_{J}$ such that $\operatorname{ad}_{H_{2}}\left(S_{J}\right)=B^{\text {non }}$. After several such transformations, we obtain the new Hamiltonian

$$
H^{(1)}\left(Z_{1}, \bar{Z}_{1}\right)=H_{2}+\sum_{j=3}^{J} H_{j}^{\mathrm{res}}+R_{J+1}^{(1)},
$$

where $R_{J+1}^{(1)}$ is the Taylor remainder term.

In Sections 3.2 and 3.3 below we will carry out two normal-form transformations to eliminate all cubic and quartic nonresonant terms in the Hamiltonian.

\subsection{Third-order Birkhoff normal form}

Our result for the third-order resonances is the same as that in the water-wave problem (Craig \& Worfolk [2]).

Proposition 4 There are no third-order resonances in the Kelvin-Helmholtz problem.

Proof: Because of conservation of momentum each monomial $Z^{P} \bar{Z}^{Q}$ appearing in the Hamiltonian satisfies $\langle P-Q, k\rangle=\sum_{k>0}(p(k)-q(k)) k=0$. It is easy to verify that there are no solutions of the relations

$$
\langle P-Q, \omega\rangle=0, \quad\langle P-Q, k\rangle=0, \quad|P|+|Q|=3 .
$$


Following the theory in Section 3.1, we are to solve $\left\{S_{3}, H_{2}\right\}+H_{3}=0$. Since the terms involved satisfy

$$
H_{2}=\sqrt{\left(\rho-\rho_{1}\right) /\left(\rho+\rho_{1}\right)} H_{2}^{0}, \quad H_{3}=\sqrt[4]{\left(\rho-\rho_{1}\right)^{5} /\left(\rho+\rho_{1}\right)^{7}} H_{3}^{0},
$$

where the superscript 0 denotes the corresponding object for the water-wave problem (Craig \& Worfolk [2]), it follows that

$$
S_{3}=\sqrt[4]{\left(\rho-\rho_{1}\right)^{3} /\left(\rho+\rho_{1}\right)^{5}} S_{3}^{0} .
$$

The formula for $S_{3}^{0}$ is given by Craig [10, equation (38)], who analysed the resulting canonical transformation as a mapping of a scale of Banach spaces; the properties described there also apply to $S_{3}$.

\subsection{Fourth-order Birkhoff normal form}

Using (32) and the fact that $H_{3}=-\left\{S_{3}, H_{2}\right\}$, we find that the quartic term in the transformed Hamiltonian $H^{(1)}$ is

$$
\begin{aligned}
H_{4}^{(1)} & =H_{4}+\frac{1}{2}\left\{S_{3}, H_{3}\right\}+\left\{S_{4}, H_{2}\right\} \\
& =\left[H_{4}+\frac{1}{2}\left\{S_{3}, H_{3}\right\}\right]^{\mathrm{res}} .
\end{aligned}
$$

The expression $\frac{1}{2}\left\{S_{3}^{0}, H_{3}^{0}\right\}+H_{4}^{0}=B_{4}^{0}$ is calculated by Craig \& Worfolk [2] and $\left\{S_{3}, H_{3}\right\}=\left(\rho-\rho_{1}\right)^{2} /\left(\rho+\rho_{1}\right)^{3}\left\{S_{3}^{0}, H_{3}^{0}\right\}$; hence

$$
\begin{aligned}
H_{4}^{(1)} & =\left[H_{4}-\frac{\left(\rho-\rho_{1}\right)^{2}}{\left(\rho+\rho_{1}\right)^{3}} H_{4}^{0}+\frac{\left(\rho-\rho_{1}\right)^{2}}{\left(\rho+\rho_{1}\right)^{3}} B_{4}^{0}\right]^{\mathrm{res}} \\
& =\left[\frac{4 \rho \rho_{1}}{\left(\rho+\rho_{1}\right)^{3}} H_{4}^{0}+A_{4}+\frac{\left(\rho-\rho_{1}\right)^{2}}{\left(\rho+\rho_{1}\right)^{3}} B_{4}^{0}\right]^{\mathrm{res}} .
\end{aligned}
$$

The next step is to calculate the right-hand side of (33) explicitly. There are two types of contributions, from the two classes of fourth-order resonances.

Proposition 5 Each resonant monomial $Z^{P} \bar{Z}^{Q}$ with

$$
|P|+|Q|=4, \quad\langle P-Q, \omega\rangle=0, \quad\langle P-Q, k\rangle=0
$$

is either generic with $P=Q$ or of the Benjamin-Feir form

$$
P=b a^{2}+b\left(a^{2}+a+1\right)^{2}, \quad Q=b(a+1)^{2}+b a^{2}(a+1)^{2}
$$


or vice versa. Generic and Benjamin-Feir resonances have frequency structures $(p: p: q: q)$ and $\left(a: a+1: a(a+1): a^{2}+a+1\right)$ respectively.

The contribution to $H_{4}^{(1)}$ from the fourth-order generic resonances is given by

$$
\begin{aligned}
H_{4}^{G}= & \frac{\rho^{2}+\rho_{1}^{2}}{\left(\rho+\rho_{1}\right)^{3}}\left(\sum_{k>0}-\frac{k^{3}}{8 \pi}\left(I_{1}^{2}(k)-3 I_{2}^{2}(k)\right)+\sum_{0<k<\ell} \frac{k^{2} \ell}{\pi} I_{2}(k) I_{2}(\ell)\right) \\
& +\frac{\rho \rho_{1}}{\pi\left(\rho+\rho_{1}\right)^{3}} \sum_{0<k<\ell}\left(k^{5 / 2} \ell^{1 / 2}-k^{3 / 2} \ell^{3 / 2}\right) I_{1}(k) I_{1}(\ell),
\end{aligned}
$$

where

$$
I_{1}(k)=\frac{1}{2}(z(k) \bar{z}(k)+w(k) \bar{w}(k)), \quad I_{2}(k)=\frac{1}{2 \mathrm{j}}(z(k) \bar{w}(k)-\bar{z}(k) w(k)),
$$

and the contribution from the Benjamin-Feir resonances is given by

$$
\begin{aligned}
H_{4}^{B-F}= & \frac{\rho \rho_{1}}{8 \pi\left(\rho+\rho_{1}\right)^{3}} \sum_{S} b^{3} a^{3}(a+1)^{3}\left(a^{2}+a+1\right)^{3 / 2} \\
& \times\left(\left(z\left(k_{1}\right) z\left(k_{4}\right)+w\left(k_{1}\right) w\left(k_{4}\right)\right)\left(\bar{z}\left(k_{2}\right) \bar{z}\left(k_{3}\right)-\bar{w}\left(k_{2}\right) \bar{w}\left(k_{3}\right)\right)\right. \\
& +\left(\bar{z}\left(k_{1}\right) \bar{z}\left(k_{4}\right)+\bar{w}\left(k_{1}\right) \bar{w}\left(k_{4}\right)\right)\left(z\left(k_{2}\right) z\left(k_{3}\right)-w\left(k_{2}\right) w\left(k_{3}\right)\right) \\
& +\left(z\left(k_{1}\right) w\left(k_{4}\right)-w\left(k_{1}\right) z\left(k_{4}\right)\right)\left(\bar{z}\left(k_{2}\right) \bar{w}\left(k_{3}\right)+\bar{w}\left(k_{2}\right) \bar{z}\left(k_{3}\right)\right) \\
& \left.+\left(\bar{z}\left(k_{1}\right) \bar{w}\left(k_{4}\right)-\bar{w}\left(k_{1}\right) \bar{z}\left(k_{4}\right)\right)\left(z\left(k_{2}\right) w\left(k_{3}\right)+w\left(k_{2}\right) z\left(k_{3}\right)\right)\right),
\end{aligned}
$$

where $S=\left\{\left(k_{1}, k_{2}, k_{3}, k_{4}\right)=\left(b a^{2}, b(a+1)^{2}, b a^{2}(a+1)^{2}, b\left(a^{2}+a+1\right)^{2}\right): a, b>0\right\}$. The above computations were done by hand and checked by computer using Mathematica.

Theorem 6 After two (formal) normal-form transformations, the KelvinHelmholtz Hamiltonian is given by the formula

$$
H^{(1)}=H_{2}+\left(H_{4}^{G}+H_{4}^{B-F}\right)+R_{5}^{(1)}
$$

where $H_{4}^{G}$ and $H_{4}^{B-F}$ are given by (35), (36) and $R_{5}^{(1)}$ is the fifth-order Taylor remainder term.

We note that $H_{4}^{B-F}$ does not vanish, in contrast to the case of the water-wave problem. In particular the quantities $I_{1}(k)$ and $I_{2}(k)$ do not commute with the Birkhoff normal-form Hamiltonian

$$
\tilde{H}=H_{2}+\left(H_{4}^{G}+H_{4}^{B-F}\right) .
$$


In the next section we will study the dynamical system with this Hamiltonian $\tilde{H}$, namely

$$
\partial_{t} z(k)=-2 \mathrm{j} \partial_{\bar{z}(k)} \tilde{H}, \quad \partial_{t} w(k)=-2 \mathrm{j} \partial_{\bar{w}(k)} \tilde{H}
$$

\subsection{Integrable subsystems of the fourth-order normal form}

We restrict our attention to the standing wave problem, for which we set $w(k)=0$ for all $k>0$. Introduce symplectic polar coordinates

$$
I_{1}(k)=\frac{1}{2} z(k) \bar{z}(k), \quad \varphi_{1}(k)=-\frac{1}{2} \arctan \left(\frac{\operatorname{im} z^{2}(k)}{\operatorname{re~} z^{2}(k)}\right) \text {, }
$$

in terms of which the fourth-order Birkhoff normal-form Hamiltonian is

$$
\begin{aligned}
\tilde{H}= & H_{2}+\left(H_{4}^{G}+H_{4}^{B-F}\right) \\
= & \sum_{k>0} \omega(k) I_{1}(k)-\frac{\rho^{2}+\rho_{1}^{2}}{\pi\left(\rho+\rho_{1}\right)^{3}} \sum_{k>0} \frac{k^{3}}{8} I_{1}^{2}(k) \\
& +\frac{\rho \rho_{1}}{\pi\left(\rho+\rho_{1}\right)^{3}} \sum_{0<k<\ell}\left(k^{5 / 2} \ell^{1 / 2}-k^{3 / 2} \ell^{3 / 2}\right) I_{1}(k) I_{1}(\ell) \\
& +\frac{\rho \rho_{1}}{\pi\left(\rho+\rho_{1}\right)^{3}} \sum_{S} b^{3} a^{3}(a+1)^{3}\left(a^{2}+a+1\right)^{3 / 2} \times \\
& \sqrt{I_{1}\left(k_{1}\right) I_{1}\left(k_{2}\right) I_{1}\left(k_{3}\right) I_{1}\left(k_{4}\right)} \cos \left(\varphi\left(k_{1}\right)-\varphi\left(k_{2}\right)-\varphi\left(k_{3}\right)+\varphi\left(k_{4}\right)\right) ;
\end{aligned}
$$

Hamilton's equations are

$$
\partial_{t} \varphi_{1}(k)=\partial_{I_{1}(k)} \tilde{H}, \quad \partial_{t} I_{1}(k)=-\partial_{\varphi_{1}(k)} \tilde{H} .
$$

In the water-wave limit $\rho_{1}=0, \rho=1$ this Hamiltonian Poisson commutes with the complete set of integrals $\left\{I_{1}(k)\right\}_{k>0}$ (see Craig \& Worfolk [2] for this result and for a discussion of the complete integrability of the flow under periodic boundary conditions). This is no longer the case for the Kelvin-Helmholtz Hamiltonian (39). There are, however, a large number of special solutions to the system (40), given by the Hamiltonian flow of (39) on the resonant subspaces

$$
R(b, a)=\left\{z: z(k)=0, k \notin\left\{b a^{2}, b(a+1)^{2}, b a^{2}(a+1)^{2}, b\left(a^{2}+a+1\right)^{2}\right\}\right\} .
$$

Theorem 7 The resonant subspaces $R(b, a)$ are invariant under the Hamiltonian flow of (39). 
Proof: In order that a coordinate subspace $L=\{z: z(k)=0, k \notin I\}$ with index set $I$ be invariant under the Hamiltonian flow of (39), the components $\left.\partial_{\bar{z}(\ell)} \tilde{H}\right|_{z \in L}$ must vanish for all $\ell \notin I$. This will be the case if every term of the Hamiltonian $\tilde{H}$ depends either not at all, or else at least quadratically, on the variables $\{(z(k), \bar{z}(k)): k \notin I\}$.

Consider the resonant coordinate subspace given by the index set

$$
I(b, a)=\left\{b a^{2}, b(a+1)^{2}, b a^{2}(a+1)^{2}, b\left(a^{2}+a+1\right)^{2}\right\} .
$$

Any generic resonant monomial which has at least one index $k \notin I(b, a)$ is at least quadratic in $(z(k), \bar{z}(k))$. To complete the proof that $\left.\partial_{\bar{z}(\ell)} \tilde{H}\right|_{z \in L}=0$ for each $\ell \notin I(a, b)$, it suffices to show that no two distinct Benjamin-Feir quartets have three wave numbers in common. Suppose ad absurdo that three of the four wave numbers of $I(a, b)$ agree with three of the four wave numbers in another Benjamin-Feir quartet $I(p, q)$. Taking the ordering into account, one finds that there are ten possibilities for this to occur. A typical case is $b a^{2}=p(q+1)^{2}, b a^{2}(a+1)^{2}=p q^{2}(q+1)^{2}$ and $b\left(a^{2}+a+1\right)^{2}=p\left(q^{2}+q+1\right)^{2}$. The ratio of the first two equalities implies that $q=a+1$, so that $b / p=$ $(a+2)^{2} / a^{2}$. The third equality implies in turn that $\sqrt{b}\left(a^{2}+1\right)=\sqrt{p} q^{2}$, whence $b / p=\left((a+1)^{2} /\left(a^{2}+1\right)\right)^{2}$. It follows that $a(a+1)^{2}=(a+2)\left(a^{2}+1\right)$, which is a contradiction. All other cases are treated in a similar fashion.

Theorem 8 The Hamiltonian flow of (39), restricted to a resonant subspace $R(b, a)$, is completely integrable.

Proof: Theorem 7 states that the flow of $\tilde{H}$ leaves $R(b, a)$ invariant, and we will now produce a sufficient number of Poisson commuting integrals. Let $N$ be a vector orthogonal to all integer vectors $P-Q$ formed from a resonance relation for the problem. Clearly $\left(N, I_{1}\right)=\sum_{k} n(k) I_{1}(k)$ is an integral, since $\left\{H_{2},\left(N, I_{1}\right)\right\}=\left(N,\left\{H_{2}, I_{1}\right\}\right)=0$, and for any resonant monomial $z^{P} \bar{z}^{Q}$ we find that $\left\{\left(N, I_{1}\right), z^{P} \bar{z}^{Q}\right\}=\left(N,\left\{I_{1}, z^{P} \bar{z}^{Q}\right\}\right)=-\mathrm{j}\langle N, P-Q\rangle z^{P} \bar{z}^{Q}=0$. Within a resonant subspace of dimension $2 n$, the number of independent vectors $N$ of the above type is $n-r$, where $r$ is the number of independent resonance relations $\langle P-Q, \omega\rangle=0$. Each Benjamin-Feir resonant subspace $R(b, a)$ has only one resonance relation, given by (34). The Hamiltonian makes up the remaining integral and is independent wherever its gradient is nonzero, since it depends nontrivially on the angular variables $\varphi_{1}(k)$.

The remainder of this paper concentrates on the structure of the flow of $\tilde{H}$ restricted to the resonant subspaces $R(b, a)$. For such questions it suffices for reasons of scale to take $b=1$. 


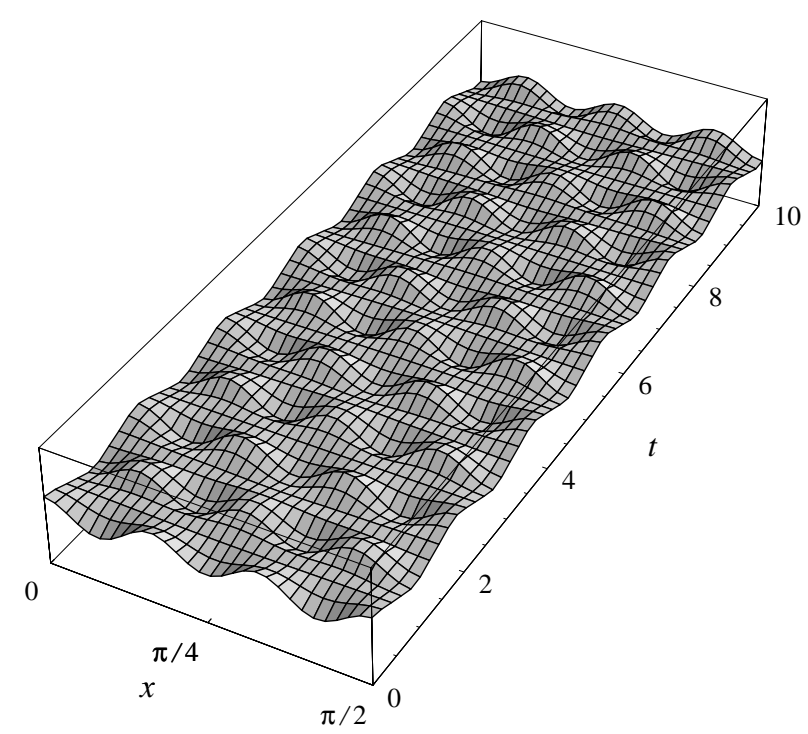

Fig. 1. Fluid motion generated by the basic periodic orbit in the $z(4)$ coordinate plane

\section{The $(1: 2: 3)$ resonant subsystem}

\subsection{The structure of $R(1,1)$}

For $a=1$ two frequencies of a Benjamin-Feir resonant quartet coincide, and the resulting subsystem has three degrees of freedom. There are two integrals of motion in addition to the Hamiltonian, and the phase space decomposes via a singular foliation into components: a two dimensional reduced phase space, and the two remaining cyclic degrees of freedom. Most orbits lie on three dimensional tori; however at singular points of the foliation and at critical points of the reduced Hamiltonian orbits lie on lower dimensional tori. In certain specific instances these are unstable, possessing one hyperbolic normal degree of freedom, with one dimensional stable and one dimensional unstable manifolds connected through one or several homoclinic orbits. Indeed there is a range of $r$ for which some of the basic periodic orbits are unstable in this manner. We will describe all of the orbits of this integrable subsystem in detail.

There are three basic families of periodic orbits which involve only one of the three available modes (two of these are guaranteed by the Lyapunov centre theorem). Restricting (38) to the $z(1)$ coordinate plane, one finds that

$$
\partial_{t} z(1)=-2 \mathrm{j}\left(\omega(1)-\frac{\rho^{2}+\rho_{1}^{2}}{8 \pi\left(\rho+\rho_{1}\right)^{3}}|z(1)|^{2}\right) z(1)
$$


which describes a family of periodic orbits as circles traversed with frequency

$$
\Omega_{1}\left(|z(1)|^{2}\right)=\omega(1)-\frac{\rho^{2}+\rho_{1}^{2}}{8 \pi\left(\rho+\rho_{1}\right)^{3}}|z(1)|^{2} .
$$

The $z(4)$ and $z(9)$ coordinate planes are similarly filled by periodic orbits with frequencies

$$
\begin{gathered}
\Omega_{4}\left(|z(4)|^{2}\right)=2 \omega(1)-\frac{8\left(\rho^{2}+\rho_{1}^{2}\right)}{\pi\left(\rho+\rho_{1}\right)^{3}}|z(4)|^{2}, \\
\Omega_{9}\left(|z(9)|^{2}\right)=3 \omega(1)-\frac{729\left(\rho^{2}+\rho_{1}^{2}\right)}{8 \pi\left(\rho+\rho_{1}\right)^{3}}|z(9)|^{2} .
\end{gathered}
$$

Note that the action-frequency maps are nondegenerate.

The fluid interface generated by any solution to the present Hamiltonian system can be computed by applying the inverse normal-form transformations to the coordinate functions $z(1), z(4), z(9)$; note that it is consistent to approximate the interface using cubic truncations of the inverse transformations. Figure 1 shows the fluid interface generated by the basic periodic orbit in the $z(4)$ coordinate plane. This solution, which has just one mode in the normalform coordinates, corresponds to a solution in the physical coordinates which is excited in three modes (modes 4, 8 and 12).

For notational convenience let us write $I_{1}(k)=I_{k}, \varphi_{1}(k)=\varphi_{k}, k=1,4,9$. Equation (39) shows that the restriction of the Hamiltonian $\tilde{H}$ to $R(1,1)$ is

$$
\begin{aligned}
\tilde{H}_{1,1}= & \omega(1)\left(I_{1}+2 I_{4}+3 I_{9}\right)-\frac{\rho^{2}+\rho_{1}^{2}}{8 \pi\left(\rho+\rho_{1}\right)^{3}}\left(I_{1}^{2}+64 I_{4}^{2}+729 I_{9}^{2}\right) \\
& -\frac{6 \rho \rho_{1}}{\pi\left(\rho+\rho_{1}\right)^{3}}\left(I_{1} I_{4}+4 I_{1} I_{9}+20 I_{4} I_{9}\right) \\
& +\frac{24 \sqrt{3} \rho \rho_{1}}{\pi\left(\rho+\rho_{1}\right)^{3}} \sqrt{I_{1} I_{9}} I_{4} \cos \left(\varphi_{1}-2 \varphi_{4}+\varphi_{9}\right) .
\end{aligned}
$$

The next structure of interest is the subspace $\left\{I_{4}=0\right\}$, which is easily seen from (40), (42) to be invariant under the Hamiltonian flow of $\tilde{H}_{1,1}$. The two action variables $I_{1}, I_{9}$ act as integrals, and the subspace is foliated by twodimensional tori which are invariant under the flow. The action-frequency map is nondegenerate; writing $I^{\prime}=\left(I_{1}, I_{9}\right)$, one finds that its derivative is

$$
\left.\partial_{I^{\prime}}^{2} \tilde{H}_{1,1}\right|_{I_{4}=0}=-\frac{\rho^{2}+\rho_{1}^{2}}{4 \pi\left(\rho+\rho_{1}\right)^{3}}\left(\begin{array}{cc}
1 & 0 \\
0 & 729
\end{array}\right)-\frac{6 \rho \rho_{1}}{\pi\left(\rho+\rho_{1}\right)^{3}}\left(\begin{array}{cc}
0 & 4 \\
4 & 0
\end{array}\right),
$$




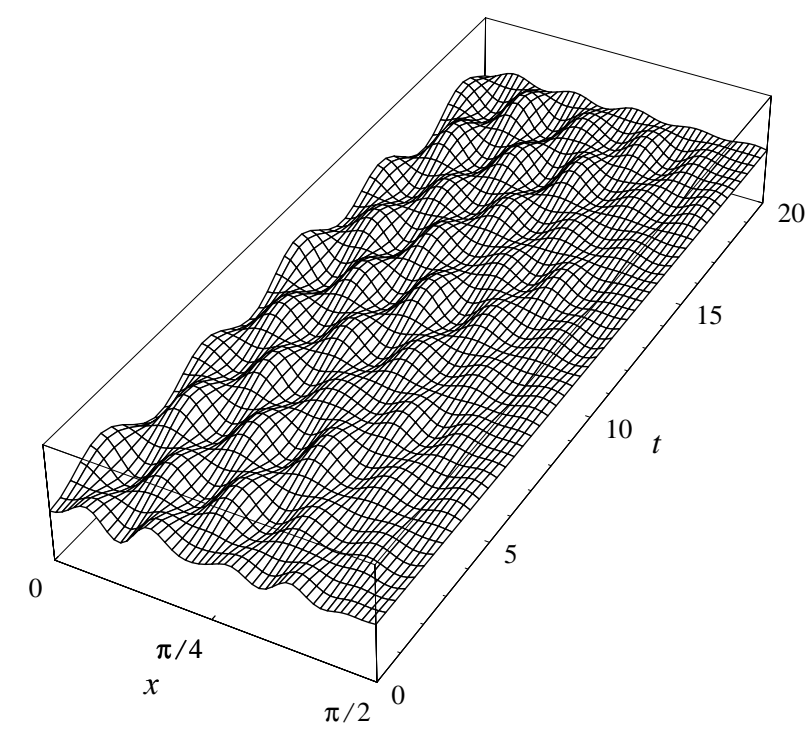

Fig. 2. Fluid motion generated by a quasiperiodic orbit in the subspace $\left\{I_{4}=0\right\}$; the interface is plotted over one half of its spatial period

which is nonzero for all $0 \leq \rho_{1}<\rho \leq 1$. The corresponding fluid interface is shown in Figure 2; the solution is excited in twelve modes (modes 1, 2, 3, 7, $8,9,10,11,17,18,19,27)$ in physical coordinates.

The rest of the system is conveniently described in rotating coordinates; we perform the simultaneous rotation

$$
\left(I_{1}, I_{4}, I_{9}\right)^{T}=R\left(L_{1}, L_{2}, L_{3}\right)^{T}, \quad\left(\varphi_{1}, \varphi_{4}, \varphi_{9}\right)^{T}=R\left(\psi_{1}, \psi_{2}, \psi_{3}\right)^{T},
$$

where

$$
R=\left(\begin{array}{ccc}
\frac{1}{\sqrt{6}} & \frac{4}{\sqrt{21}} & \frac{1}{\sqrt{14}} \\
\frac{-2}{\sqrt{6}} & \frac{1}{\sqrt{21}} & \frac{2}{\sqrt{14}} \\
\frac{1}{\sqrt{6}} & \frac{-2}{\sqrt{21}} & \frac{3}{\sqrt{14}}
\end{array}\right)
$$

Because $I_{k} \geq 0$ we must take $L_{3} \geq 0,-2 \sqrt{2} L_{3} / 3 \sqrt{3} \leq L_{2} \leq 4 \sqrt{2} L_{3} / \sqrt{3}$ and $L_{1} \in\left[L_{1}^{\min }, L_{1}^{\max }\right]$, where

$$
\begin{gathered}
L_{1}^{\min }=\sqrt{6} \max \left\{\left(-\frac{4}{\sqrt{21}} L_{2}-\frac{1}{\sqrt{14}} L_{3}\right),\left(\frac{2}{\sqrt{21}} L_{2}-\frac{3}{\sqrt{14}} L_{3}\right)\right\}, \\
L_{1}^{\max }=\sqrt{6}\left(\frac{1}{2 \sqrt{21}} L_{2}+\frac{1}{\sqrt{14}} L_{3}\right) .
\end{gathered}
$$

Note that $L_{1}=L_{1}^{\max }$ occurs when $I_{4}=0$ and $L_{1}=L_{1}^{\min }$ occurs when either $I_{1}=0$ or $I_{9}=0$. 
The Hamiltonian (42) depends only on the single angle $\varphi_{1}-2 \varphi_{4}+\varphi_{9}=\sqrt{6} \psi_{1}$. The quantities $L_{2}$ and $L_{3}$ are therefore independent integrals of motion and the system (40) reduces to a system with one degree of freedom, namely

$$
\partial_{t} L_{1}=-\partial_{\psi_{1}} \tilde{H}_{1,1}, \quad \partial_{t} \psi_{1}=\partial_{L_{1}} \tilde{H}_{1,1}
$$

the angles $\psi_{2}, \psi_{3}$ are recovered by quadrature. The reduced phase space $P\left(L_{2}, L_{3}\right)$ is topologically a sphere, and has canonical coordinates $\left(L_{1}, \psi_{1}\right)$, where $L_{1}$ lies in the above range and $\sqrt{6} \psi_{1} \in[0,2 \pi)$. The reduced phase spaces form a singular foliation of $R(1,1)$ whose leaves are the intersections of the level sets of the positive-definite quadratic form $L_{3}=1 / \sqrt{14}\left(|z(1)|^{2}+2|z(4)|^{2}+\right.$ $\left.3|z(9)|^{2}\right)$ and the quadratic form $L_{2}=1 / \sqrt{21}\left(4|z(1)|^{2}+|z(4)|^{2}-2|z(9)|^{2}\right)$. The singular leaves are extrema of such intersections.

Most orbits of (44) on the reduced phase space $P\left(L_{2}, L_{3}\right)$ are closed; the associated solutions of (38) therefore lie on three-dimensional tori in $R(1,1)$ and are typically quasi-periodic. At critical points $\left(L_{1}, \psi_{1}\right)$ where $\left(\partial_{L_{1}} \tilde{H}_{1,1}, \partial_{\psi_{1}} \tilde{H}_{1,1}\right)=0$ this is no longer the case, and the solution lies on a lower dimensional torus in $R(1,1)$. For every choice of the integrals $L_{2}, L_{3}$ the reduced phase space $P\left(L_{2}, L_{3}\right)$ intersects the coordinate subspace $\left\{I_{4}=0\right\}$ discussed above in a two dimensional torus. When $\sqrt{6} L_{2}=L_{3}\left(I_{1}=I_{9}\right), P\left(L_{2}, L_{3}\right)$ is a singular leaf of the foliation of $R(1,1)$; in this case $P\left(L_{2}, L_{3}\right)$ also intersects the $z(4)$ coordinate plane in a basic periodic orbit.

\subsection{Regular leaves}

An explicit calculation shows that the system of equations (44) on the reduced phase space are

$$
\begin{aligned}
\partial_{t} L_{1}= & \frac{72 \sqrt{2}}{\pi} \frac{\rho \rho_{1}}{\left(\rho+\rho_{1}\right)^{3}} I_{4} \sqrt{I_{1} I_{9}} \sin \left(\sqrt{6} \psi_{1}\right) \\
\partial_{t} \psi_{1}= & -\frac{\rho^{2}+\rho_{1}^{2}}{8 \sqrt{6} \pi\left(\rho+\rho_{1}\right)^{3}}\left(2 I_{1}-256 I_{4}+1458 I_{9}\right) \\
& -\frac{\sqrt{6} \rho \rho_{1}}{\pi\left(\rho+\rho_{1}\right)^{3}}\left(2 I_{1}+21 I_{4}-36 I_{9}\right) \\
& +\frac{24}{\sqrt{2} \pi} \frac{\rho \rho_{1}}{\left(\rho+\rho_{1}\right)^{3}}\left(\frac{\frac{1}{2} I_{1} I_{4}+\frac{1}{2} I_{4} I_{9}-2 I_{1} I_{9}}{\sqrt{I_{1} I_{9}}}\right) \cos \left(\sqrt{6} \psi_{1}\right),
\end{aligned}
$$

where $I_{k}=I_{k}\left(L_{1}, L_{2}, L_{3}\right)$ is given by (43). The reduced phase space has coordinate singularities at $L_{1}=L_{1}^{\min }\left(\right.$ either $I_{1}=0$ or $I_{9}=0$ ) and at $L_{1}=L_{1}^{\max }$ $\left(I_{4}=0\right)$. The former corresponds to a pole of the sphere, $\left\{L_{1}=L_{1}^{\min }\right\}$ is 


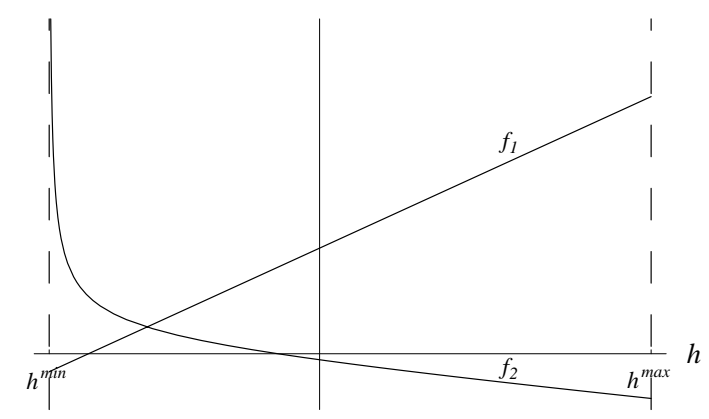

Fig. 3. The graphs of $f_{1}, f_{2}$

identified as a point, and the vector field (45), (46) is smooth. The latter set $\left\{L_{1}=L_{1}^{\max }\right\}$ is also identified as a pole, which however is a conic singularity of the sphere.

Zeros of the vector field (45), (46) on the reduced phase space can be divided into three cases, according to whether the right-hand side of (45) vanishes (a) when $\sin \left(\sqrt{6} \psi_{1}\right)=0$, (b) when $I_{4}=0$ or (c) when either $I_{1}=0$ or $I_{9}=0$ (they do not simultaneously vanish on a regular leaf). Let us immediately note that case (c) zeros do not occur, since $I_{1}=0$ or $I_{9}=0$ corresponds to the regular pole $L_{1}=L_{1}^{\min }$ of the sphere.

In case (a) the zeros of (45), (46) are critical points of $\tilde{H}_{1,1}$ on the reduced phase space. These occur only at $\left(L_{1}, n \pi / \sqrt{6}\right)$, where $n \in \mathbb{Z}$ and $L_{1}$ satisfies the equation

$$
\begin{aligned}
\frac{r}{8}\left(2 I_{1}-256 I_{4}\right. & \left.+1458 I_{9}\right)+6\left(2 I_{1}+21 I_{4}-36 I_{9}\right) \\
& =(-1)^{n} 24 \sqrt{3}\left(\frac{\frac{1}{2} I_{1} I_{4}+\frac{1}{2} I_{4} I_{9}-2 I_{1} I_{9}}{\sqrt{I_{1} I_{9}}}\right) .
\end{aligned}
$$

Introduce homogeneous coordinates $h=L_{1} / L_{3}$ and $g=L_{2} / L_{3}$ and define functions $f_{1}, f_{2}$ by $f_{1}(h)=$ L.H.S. $(47) \times L_{3}, f_{2}(h)=$ R.H.S. $(47) \times(-1)^{n} L_{3}$; note that $f_{1}$ and $f_{2}$ depend parametrically on $g$. Critical points of $\tilde{H}_{1,1}$ correspond to solutions of $f_{1}(h)=(-1)^{n} f_{2}(h)$. The stability of a critical point of $\tilde{H}_{1,1}$ is determined by the spectrum of $J \partial_{\left(L_{1}, \psi_{1}\right)}^{2} \tilde{H}_{1,1}$. In our case (a), we have that $\partial^{2} \tilde{H}_{1,1} / \partial L_{1} \partial \psi_{1}=0$ and $\operatorname{sign}\left(\partial^{2} \tilde{H}_{1,1} / \partial \psi_{1}^{2}\right)=(-1)^{n+1}$. The critical point is therefore elliptic if $(-1)^{n} \operatorname{sign}\left(\partial^{2} \tilde{H}_{1,1} / \partial L_{1}^{2}\right)<0$ and hyperbolic otherwise. This criterion is related to $f_{1}, f_{2}$ by the fact that $\operatorname{sign}\left(\partial^{2} \tilde{H}_{1,1} / \partial L_{1}^{2}\right)=$ $\operatorname{sign}\left(-f_{1}^{\prime}(h)+(-1)^{n} f_{2}^{\prime}(h)\right)$.

Elementary calculations show that $f_{2}$ is decreasing and convex and $f_{1}$ is affine linear and increasing; moreover $f_{1}\left(h^{\max }\right)>0, f_{2}\left(h^{\max }\right)<0, f_{1}(h) \rightarrow+\infty$ as $h \downarrow h^{\mathrm{min}}$. It follows that when $n$ is even there is precisely one intersection of the graphs of $f_{1}$ and $f_{2}$ and hence a critical point of $\tilde{H}_{1,1}$. This critical 


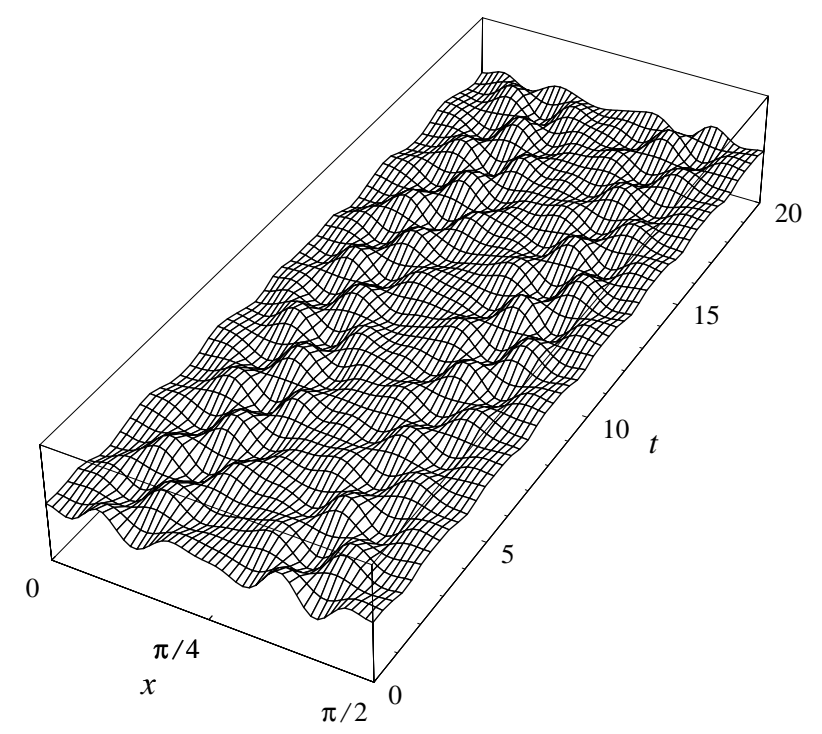

Fig. 4. Fluid motion generated by an elliptic critical point of $\tilde{H}_{1,1}$; the interface is plotted over one half of its spatial period

point is elliptic since $(-1)^{n} \operatorname{sign}\left(-f_{1}^{\prime}(h)+(-1)^{n} f_{2}^{\prime}(h)\right)<0$ (see Figure 3); the corresponding fluid interface is shown in Figure 4; the solution is excited in nineteen modes $(1,2,3,4,5,6,7,8,9,10,11,12,13,14,17,18,19,22,27)$ in physical coordinates. Similar considerations may be applied when $n$ is odd. One finds that $f_{1}$ and $-f_{2}$ do not intersect for small $r$. However there is a constant $r_{0}>2$ and, for each $r>r_{0}$, an interval $(b(r), t(r))$ such that $f_{1}$ and $-f_{2}$ intersect in two points when $g \in(b(r), t(r))$. Comparing the slopes of the two graphs at their intersections and using the above sign criterion, one finds that one of the resulting critical points is elliptic and one is hyperbolic. The Lyapunov exponent of the hyperbolic critical point is

$$
\begin{aligned}
& \Lambda_{1}=\frac{1}{\left(\rho+\rho_{1}\right)^{3} \pi} \sqrt{144 \rho \rho_{1} I_{4} \sqrt{3 I_{1} I_{9}}} \\
& \times\left(\frac{493}{12}\left(\rho^{2}+\rho_{1}^{2}\right)-76 \sqrt{6} \rho \rho_{1}-\rho \rho_{1} \sqrt{\frac{3}{I_{1}^{3} I_{9}^{3}}}\left(8 I_{1} I_{9}^{2}+8 I_{1}^{2} I_{9}+I_{4}\left(I_{1}-I_{9}\right)^{2}\right)\right)^{\frac{1}{2}} .
\end{aligned}
$$

Zeros of the vector field (45), (46) at the coordinate singularities in case (b) correspond to the existence of stable and unstable manifolds to tori in the invariant set $\left\{I_{4}=0\right\}$; when such zeros exist this set contains hyperbolic 'whiskered' tori. Case (b) zeros are of the form $\left(L_{1}^{\max }, \psi_{1}\right)$, where $\psi_{1}$ satisfies the equation

$$
\frac{r}{192 \sqrt{3}}\left(I_{1}+729 I_{9}\right)+\frac{1}{4 \sqrt{3}}\left(I_{1}-18 I_{9}\right)=-\sqrt{I_{1} I_{9}} \cos \left(\sqrt{6} \psi_{1}\right) .
$$



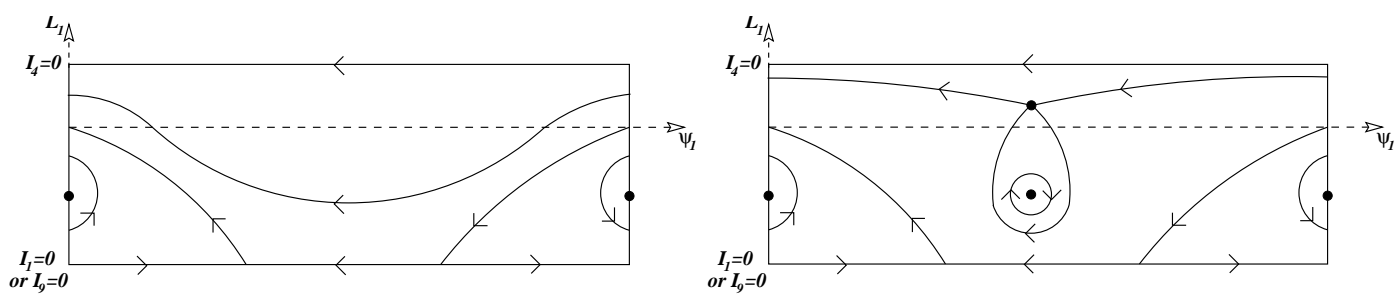

Fig. 5. Phase portrait of $P\left(L_{2}, L_{3}\right)$ (regular leaf): (a) $r<r_{0}$ or $r>r_{0}$, $g \notin\left((b(r), t(r)) ;(b) r>r_{0}, g \in(b(r), t(r))\right.$

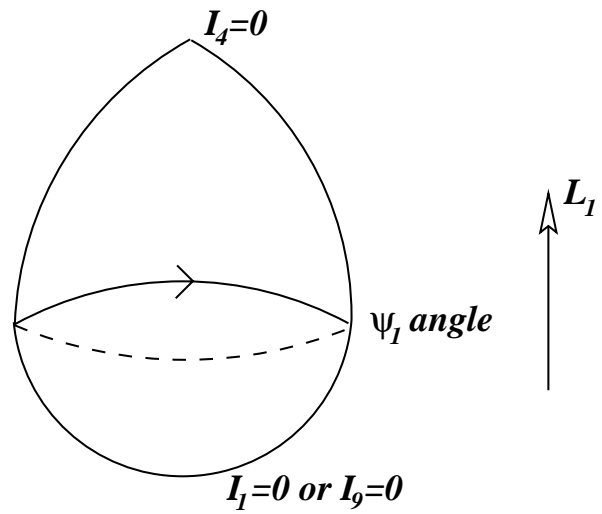

Fig. 6. A regular leaf: a sphere with a conical singularity at one pole

The left hand side of this equation lies in the range of $-\sqrt{I_{1} I_{9}} \cos \left(\sqrt{6} \psi_{1}\right)$ whenever $5 / 2-79 r / 64-27 r^{2} / 1024 \geq 0$, which occurs for $r \in\left[r_{m}, r_{M}\right]$. A direct calculation shows that $r_{M} \sim 1.96<2$, and since $r=\left(\rho^{2}+\rho_{1}^{2}\right) / \rho \rho_{1} \geq 2$ there are therefore no zeros in case (b). This implies that on a regular leaf the lower dimensional tori of the set $\left\{I_{4}=0\right\}$ are stable.

The phase portraits of $P\left(L_{2}, L_{3}\right)$ are sketched in Figure 5 . Note that the lines $L_{1}=L_{1}^{\mathrm{min}}, L_{1}=L_{1}^{\mathrm{max}}$ are coordinate singularities; the nature of a regular leaf near these points is sketched in Figure 6 . The orbit on the line $L_{1}=L_{1}^{\max }$ in Figure 5 corresponds to a two-dimensional torus in $\left\{I_{4}=0\right\}$ (see Section 4.1). The phase portraits reflect the fact that this torus is always stable.

\subsection{Singular leaves}

The reduced phase space gives a singular leaf of the foliation when $\sqrt{6} L_{2}=L_{3}$, in which case equations (45), (46) simplify to

$$
\begin{aligned}
\partial_{t} L_{1} & =\frac{72 \sqrt{2}}{\pi} \frac{\rho \rho_{1}}{\left(\rho+\rho_{1}\right)^{3}} I_{1} I_{4} \sin \left(\sqrt{6} \psi_{1}\right) \\
\partial_{t} \psi_{1} & =-\frac{\rho^{2}+\rho_{1}^{2}}{8 \sqrt{6} \pi\left(\rho+\rho_{1}\right)^{3}}\left(1460 I_{1}-256 I_{4}\right)-\frac{\sqrt{6} \rho \rho_{1}}{\pi\left(\rho+\rho_{1}\right)^{3}}\left(-34 I_{1}+21 I_{4}\right)
\end{aligned}
$$




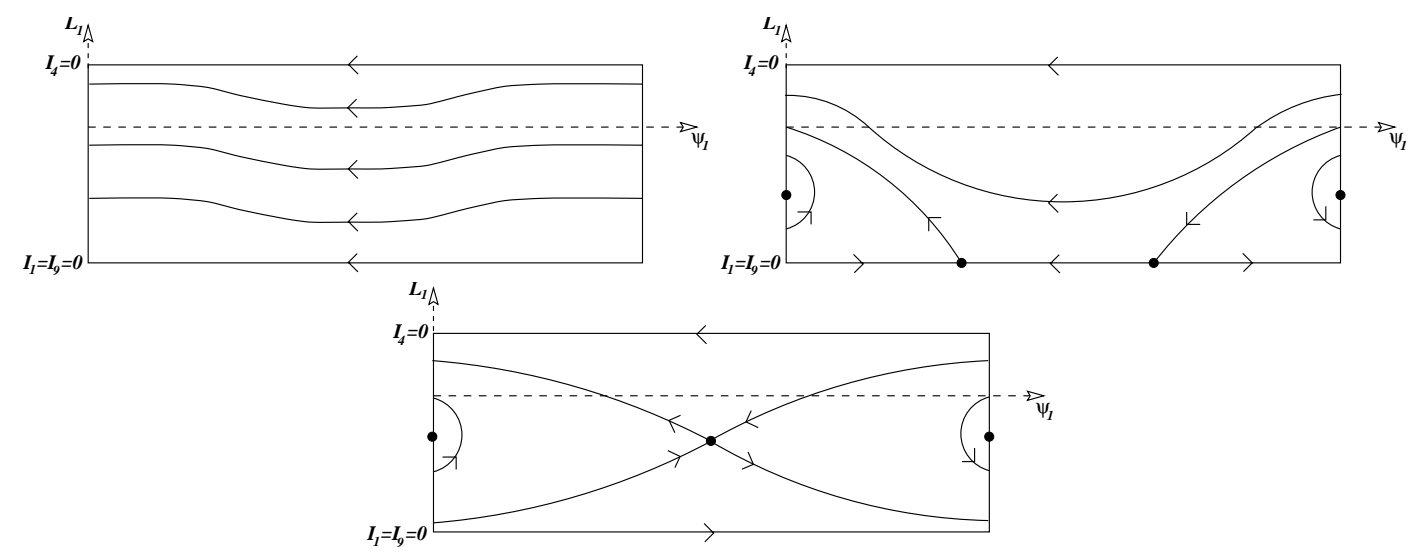

Fig. 7. Phase portrait of $P\left(L_{2}, L_{3}\right)$ (singular leaf): (a) $r<r_{0}$; (b) $r \in\left(r_{0}, r_{1}\right)$; (c) $r>r_{1}$.

$$
+\frac{24}{\sqrt{2} \pi} \frac{\rho \rho_{1}}{\left(\rho+\rho_{1}\right)^{3}}\left(I_{4}-2 I_{1}\right) \cos \left(\sqrt{6} \psi_{1}\right)
$$

note that the right-hand side of $(49)$ is linear in $L_{1}$. Zeros of the vector field (48), (49) can occur (a) when $\sin \left(\sqrt{6} \psi_{1}\right)=0$, (b) when $I_{4}=0$ or (c) when $I_{1}=I_{9}=0$. The analysis of cases (a) and (b) is similar to that in Section 4.2. In case (a) there is one elliptic critical point for $n$ even, $r>r_{0}=63 / 16-3 \sqrt{3} / 4$ and one hyperbolic critical point for $n$ odd, $r>r_{1}=63 / 16+3 \sqrt{3} / 4$; its Lyapunov exponent is

$$
\Lambda_{2}=\frac{1}{\left(\rho+\rho_{1}\right)^{3} \pi} \sqrt{144 \sqrt{3} \rho \rho_{1} I_{1} I_{4}}\left(\frac{493}{12}\left(\rho^{2}+\rho_{1}^{2}\right)-(76 \sqrt{6}+16 \sqrt{3}) \rho \rho_{1}\right)^{\frac{1}{2}} .
$$

In case (b) there are no zeros of (48), (49). Finally, case (c) zeros are of the form $\left(L_{1}^{\text {min }}, \psi_{1}\right)$, where $\cos \left(\sqrt{6} \psi_{1}\right)=(63-16 r) / 12 \sqrt{3}$. It follows that there are two such zeros for $r \in\left(r_{0}, r_{1}\right)$; they are hyperbolic with Lyapunov exponent

$$
\Lambda_{3}=\frac{24 \sqrt{3} \rho \rho_{1} I_{4}}{\left(\rho+\rho_{1}\right)^{3} \pi}\left|\sin \left(\sqrt{6} \psi_{1}\right)\right| .
$$

The phase portraits of $P\left(L_{2}, L_{3}\right)$ are sketched in Figure 7 , and its nature near the coordinate singularities at $L_{1}=L_{1}^{\min }, L_{1}=L_{1}^{\max }$ is depicted in Figure 8. As for a regular leaf, the orbit on the line $L_{1}=L_{1}^{\max }$ corresponds to a stable twodimensional torus in $\left\{I_{4}=0\right\}$. Any orbit on the line $L_{1}=L_{1}^{\text {min }}$ corresponds to a basic periodic orbit in the $z(4)$ coordinate plane. The heteroclinic orbit inside $P\left(L_{2}, L_{3}\right)$ which connects the zeros on the line $L_{1}=L_{1}^{\min }$ in Figure $7(\mathrm{~b})$ therefore represents an orbit which is homoclinic to a basic periodic orbit in the $z(4)$ coordinate plane; the fluid motion generated by this homoclinic orbit is shown in Figure 9. The above conclusions are summarised in the following proposition. 


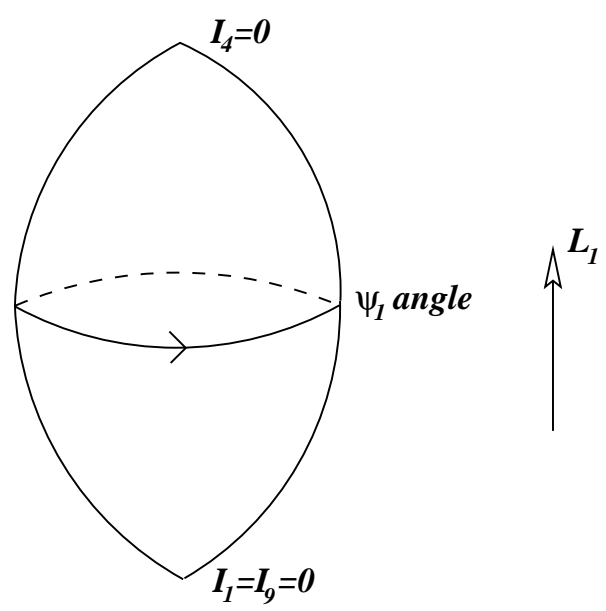

Fig. 8. A singular leaf: a sphere with conical singularities at both poles

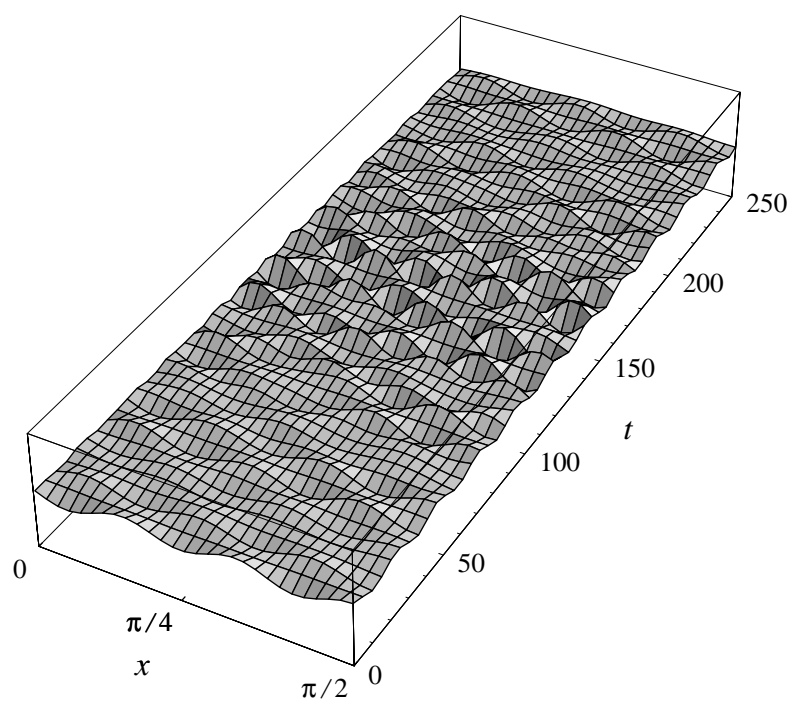

Fig. 9. Fluid motion generated by an orbit homoclinic to a basic periodic orbit in the $z(4)$ coordinate plane; the interface is plotted over one half of its spatial period

Proposition 9 When $\sqrt{6} L_{2}=L_{3}$ the reduced phase space is bounded by a two dimensional torus in the subspace $\left\{I_{4}=0\right\}$ and a periodic orbit in the $z(4)$ coordinate plane. This periodic orbit has the following stability properties.

(i) It is stable when $2<r<r_{0}$.

(ii) It loses stability when $r_{0}<r<r_{1}$. An elliptic critical point of $\tilde{H}_{1,1}$ moves off the $z(4)$ coordinate plane into the interior of $P\left(L_{2}, L_{3}\right)$. It is encircled by an orbit homoclinic to the unstable periodic orbit.

(iii) It regains stability when $r>r_{1}$. A hyperbolic critical point of $\tilde{H}_{1,1}$ moves off the $z(4)$ coordinate plane into the interior of $P\left(L_{2}, L_{3}\right)$; its stable and unstable directions are connected by two homoclinic orbits. 


\section{The $\left(a: a+1: a(a+1): a^{2}+a+1\right)$ resonant subsystems}

Our last considerations are of the system (40) restricted to the resonant subspace $R(b, a)$ for $a \geq 2$, where again it suffices to examine the case $b=1$. This is also an integrable subsystem of the fourth-order Birkhoff normal form, and we can describe the phase space and the orbits of the system in detail. Most of them lie on four dimensional invariant tori, but there are a number of singular leaves and lower dimensional tori that play an important role for the flow. In particular, certain lower dimensional tori are unstable 'whiskered' tori in a hyperbolic environment, with stable and unstable manifolds connected through one or several homoclinic orbits.

Writing for notational convenience $I_{1}\left(a^{2}\right)=I_{1}, I_{1}\left((a+1)^{2}\right)=I_{2}, I_{1}\left(a^{2}(a+\right.$ $\left.1)^{2}\right)=I_{3}$ and $I_{1}\left(\left(a^{2}+a+1\right)^{2}\right)=I_{4}\left(\right.$ with similar notation for $\varphi_{1}(k)$ and $\left.z(k)\right)$, one finds that the Hamiltonian is

$$
\begin{aligned}
& \tilde{H}_{1, a}=\omega(1)\left(a I_{1}+(a+1) I_{2}+a(a+1) I_{3}+\left(a^{2}+a+1\right) I_{4}\right) \\
& -\frac{\rho^{2}+\rho_{1}^{2}}{8 \pi\left(\rho+\rho_{1}\right)^{3}}\left(a^{6} I_{1}^{2}+(a+1)^{6} I_{2}^{2}+a^{6}(a+1)^{6} I_{3}^{2}+\left(a^{2}+a+1\right)^{6} I_{4}^{2}\right) \\
& -\frac{\rho \rho_{1}}{\pi\left(\rho+\rho_{1}\right)^{3}}\left(a^{3}(a+1)(2 a+1) I_{1} I_{2}\right. \\
& \quad+a^{7}(a+1)(a+2) I_{1} I_{3}+a(a-1)(a+1)^{7} I_{2} I_{3} \\
& \quad+a^{3}(a+1)^{2}\left(a^{2}+1\right)\left(a^{2}+a+1\right) I_{1} I_{4} \\
& \quad+a^{2}(a+1)^{3}\left(a^{2}+a+1\right)\left(a^{2}+2 a+2\right) I_{2} I_{4} \\
& \left.\quad+a^{3}(a+1)^{3}\left(a^{2}+a+1\right)\left(2 a^{2}+2 a+1\right) I_{3} I_{4}\right) \\
& \quad+\frac{\rho \rho_{1}}{4 \pi\left(\rho+\rho_{1}\right)^{3}} a^{3}(a+1)^{3}\left(a^{2}+a+1\right)^{3 / 2} \sqrt{I_{1} I_{2} I_{3} I_{4}} \cos \left(\varphi_{1}-\varphi_{2}-\varphi_{3}+\varphi_{4}\right) .
\end{aligned}
$$

There are four basic families of periodic orbits which involve only one of the four available Fourier modes. The frequency of the periodic solutions in the $z_{1}$ coordinate plane is

$$
\Omega_{1}\left(\left|z_{1}\right|^{2}\right)=\omega(1) a-\frac{\rho^{2}+\rho_{1}^{2}}{8 \pi\left(\rho+\rho_{1}\right)^{3}} a^{6}\left|z_{1}\right|^{2},
$$

which has a nondegenerate action-frequency map. The other three families have frequencies which are derived from (50) in the same manner, and they are also nondegenerate. The $\left(z_{k}, z_{\ell}\right)$ coordinate subspaces (for $k=1, \ldots 4$, $\ell=k+1, \ldots, 4)$ are invariant under the flow of (40), and each of these is foliated by a two dimensional family of two dimensional invariant tori. There are values of $r \in(2,+\infty)$ for which the action-frequency map degenerates, but for each choice of $a$, the map is nondegenerate for almost all $r$. 
The remainder of the phase space is described in rotating coordinates by using the transformation $\left(I_{1}, I_{2}, I_{3}, I_{4}\right)^{T}=R\left(L_{1}, L_{2}, L_{3}, L_{4}\right)^{T},\left(\varphi_{1}, \varphi_{2}, \varphi_{3}, \varphi_{4}\right)^{T}=$ $R\left(\psi_{1}, \psi_{2}, \psi_{3}, \psi_{4}\right)^{T}$; the orthogonal matrix $R$ is chosen so that

$$
\varphi_{1}-\varphi_{2}-\varphi_{3}+\varphi_{4}=2 \psi_{1}
$$

and

$$
\sqrt{2}\left(a^{2}+a+1\right) L_{4}=a I_{1}+(a+1) I_{2}+a(a+1) I_{3}+\left(a^{2}+a+1\right) I_{4}>0 .
$$

The Hamiltonian $\tilde{H}_{1, a}$ depends upon only one angle $\psi_{1}$, and the quantities $L_{2}, L_{3}$ and $L_{4}$ are therefore independent integrals of motion. The Hamiltonian itself provides the fourth integral of the system. The reduced phase spaces $P\left(L_{2}, L_{3}, L_{4}, a\right)$ form a singular foliation of $R(1, a)$ whose leaves are the intersections of the level sets of $L_{2}, L_{3}$ and $L_{4}$; singular leaves of the resulting foliation occur at extrema of these intersections.

Each reduced phase space is topologically a two dimensional sphere with canonical coordinates $\left(L_{1}, \psi_{1}\right)$, where $L_{1}^{-} \leq L_{1} \leq L_{1}^{+}, 0 \leq \psi_{1}<\pi$. Most orbits on $P\left(L_{2}, L_{3}, L_{4}, a\right)$ are closed; they typically give rise to quasiperiodic orbits in $R(1, a)$ with four independent frequencies. At critical points of the Hamiltonian on the reduced phase space, and possibly at coordinate singularities at the poles of these spheres, orbits will lie on lower dimensional tori.

Singular leaves of the foliation occur for coincidences $I_{1}=I_{4}, I_{2}=I_{3}$ or both. Leaves are otherwise regular. The equations of motion on $P\left(L_{2}, L_{3}, L_{4}, a\right)$ are

$$
\begin{aligned}
\partial_{t} L_{1}= & \frac{\rho \rho_{1}}{2 \pi\left(\rho+\rho_{1}\right)^{3}} a^{3}(a+1)^{3}\left(a^{2}+a+1\right)^{3 / 2} \sqrt{I_{1} I_{2} I_{3} I_{4}} \sin \left(2 \psi_{1}\right), \\
\partial_{t} \psi_{1}= & -\frac{\rho^{2}+\rho_{1}^{2}}{8 \pi\left(\rho+\rho_{1}\right)^{3}}\left(a^{6} I_{1}-(a+1)^{6} I_{2}-a^{6}(a+1)^{6} I_{3}+\left(a^{2}+a+1\right)^{6} I_{4}\right) \\
& -\frac{\rho \rho_{1}}{2 \pi\left(\rho+\rho_{1}\right)^{3}} \times \\
& \left(3 a^{5}(a+1)^{2} I_{1}+a(a+1)\left(3 a^{2}+3 a+1\right)\left(a^{3}+3 a^{2}+4 a+1\right) I_{2}\right. \\
& +a(a+1)\left(2 a^{8}+8 a^{7}+12 a^{6}+8 a^{5}+7 a^{4}+10 a^{3}+10 a^{2}+5 a+1\right) I_{3} \\
& \left.-2 a^{2}(a+1)^{2}\left(a^{2}+a+1\right)^{3} I_{4}\right) \\
& +\frac{\rho \rho_{1}}{16 \pi\left(\rho+\rho_{1}\right)^{3}} \frac{a^{3}(a+1)^{3}\left(a^{2}+a+1\right)^{3 / 2}}{\sqrt{I_{1} I_{2} I_{3} I_{4}}} \times \\
& \left(I_{2} I_{3} I_{4}-I_{1} I_{3} I_{4}-I_{1} I_{2} I_{4}+I_{1} I_{2} I_{3}\right) \cos \left(2 \psi_{1}\right),
\end{aligned}
$$

where each $I_{j}=I_{j}\left(L_{1}, L_{2}, L_{3}, L_{4}\right)$ is a linear combination of $L_{1}, L_{2}, L_{3}, L_{4}$.

On a regular leaf the sets $\left\{L_{1}=L_{1}^{ \pm}\right\}$are coordinate singularities which are 

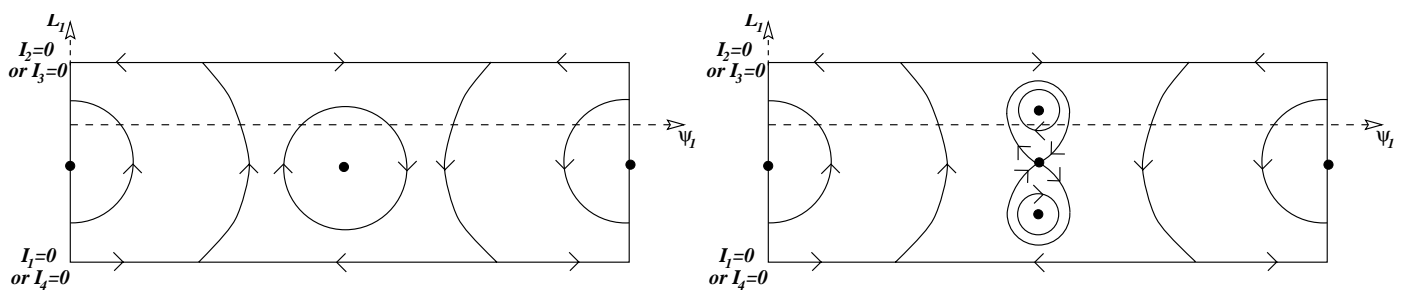

Fig. 10. The two possible phase portraits of $P\left(L_{2}, L_{3}, L_{4}, a\right)$ (regular leaf)

identified as the poles of the sphere, and the vector field is smooth through them. Critical points of $\tilde{H}_{1, a}$ occur only when $\psi_{1}=n \pi / 2$, so that the righthand side of (51) vanishes. The right-hand side of (52) also vanishes when

$$
\begin{aligned}
& r\left(a^{6} I_{1}-(a+1)^{6} I_{2}-a^{6}(a+1)^{6} I_{3}+\left(a^{2}+a+1\right)^{6} I_{4}\right) \\
& +4\left(3 a^{5}(a+1)^{2} I_{1}+a(a+1)\left(3 a^{2}+3 a+1\right)\left(a^{3}+3 a^{2}+4 a+1\right) I_{2}\right. \\
& +a(a+1)\left(2 a^{8}+8 a^{7}+12 a^{6}+8 a^{5}+7 a^{4}+10 a^{3}+10 a^{2}+5 a+1\right) I_{3} \\
& \left.-2 a^{2}(a+1)^{2}\left(a^{2}+a+1\right)^{3} I_{4}\right) \\
= & (-1)^{n} \frac{a^{3}(a+1)^{3}\left(a^{2}+a+1\right)^{3 / 2}}{2 \sqrt{I_{1} I_{2} I_{3} I_{4}}}\left(I_{2} I_{3} I_{4}-I_{1} I_{3} I_{4}-I_{1} I_{2} I_{4}+I_{1} I_{2} I_{3}\right) ;
\end{aligned}
$$

this equation takes the form $(-1)^{n} f_{1}\left(L_{1}, r\right)=f_{2}\left(L_{1}\right)$. The function $f_{1}$ is affine linear in $L_{1}$ and has positive slope for all $r$. The function $f_{2}$ is strictly decreasing and is asymptotic to $\mp \infty$ as $L_{1} \rightarrow L_{1}^{ \pm}$. It has one inflection point and is convex to the left and concave to the right of this point. There are therefore always two critical points of $\tilde{H}_{1, a}$, one each for $n$ even and $n$ odd, and both of them are elliptic. It follows from the geometric character of the graphs of $f_{1}, f_{2}$ that there are only two other possible critical points, one elliptic and one hyperbolic, and they will occur for $n$ odd. Depending upon the values of $L_{2}, L_{3}, L_{4}$ and $a$ there are cases for which the extra two critical points occur for sufficiently large $r$, and others where the extra critical points appear only for values of $r$ sufficiently close to 2; the details of these explicit calculations are not included here. Whenever these extra two critical points occur, there are also two homoclinic orbits to the hyperbolic critical point which separate the three elliptic points from each other. The two possible phase portraits are shown in Figure 10.

When $I_{1}=I_{4}$ or $I_{2}=I_{3}$ or both, the reduced phase space forms a singular leaf. The sets $\left\{L_{1}=L_{1}^{ \pm}\right\}$are coordinate singularities which are identified as the poles of the sphere. When $I_{1}=I_{4}$ there is a conical singularity at $L_{1}=L_{1}^{-}$; here the reduced phase space intersects the $\left\{I_{1}=I_{4}=0\right\}$ coordinate subspace in a two-dimensional torus. When $I_{2}=I_{3}$ there is a conical singularity at $L_{1}=L_{1}^{+}$; here the reduced phase space intersects the $\left\{I_{2}=I_{3}=0\right\}$ coordinate subspace in a two-dimensional torus. The sphere has two conical singularities in the third case. To analyse the phase portraits on singular leaves, one seeks critical points of the Hamiltonian $\tilde{H}_{1, a}$ on the reduced phase space and examines 
the coordinate singularities. Critical points $\left(L_{1}, \psi_{1}\right)$ satisfy $\psi_{1}=n \pi / 2$ and $(-1)^{n} f_{1}\left(L_{1}, r\right)=f_{2}\left(L_{1}\right)$. The inflection point of $f_{2}$ coincides with $L_{1}^{-}$when $I_{1}=I_{4}$, so that $f_{2}$ is concave. When $I_{2}=I_{3}$ the inflection point occurs at $L_{1}=L_{1}^{+}$and $f_{2}$ is convex, and when $I_{1}=I_{4}$ and $I_{2}=I_{3}$ the function $f_{2}$ is linear.

The analysis of the case $I_{2}=I_{3}$ is similar to that in Section 4.2. One finds that when $n$ is even there is one critical point on the meridian $\psi_{1}=n \pi / 2$, and when $n$ is odd there could be no critical points or there may be two, one elliptic and one hyperbolic. At the pole $L_{1}=L_{1}^{+}$the reduced phase space intersects the coordinate subspace $\left\{I_{2}=I_{3}=0\right\}$ in a two dimensional torus. A direct calculation shows that the right-hand side of (52) never vanishes at $L_{1}=L_{1}^{+}$, so that this two dimensional torus is stable for all $a \geq 2$ and for all $r \in(2,+\infty)$. The phase portrait of $P\left(L_{2}, L_{3}, L_{4}, a\right)$ is similar to that depicted in Figure 5.

The case $I_{1}=I_{4}$ is treated similarly, with a similar phase portrait and identical conclusions, although the roles of $L_{1}^{ \pm}$must be reversed. The right-hand side of (52) does, however, vanish at certain points on $L_{1}=L_{1}^{-}$, so that there are unstable 'whiskered' tori in $\left\{I_{1}=I_{4}=0\right\}$, with one dimensional stable and unstable manifolds contained in the singular leaf $P\left(L_{2}, L_{3}, L_{4}, a\right)$. The following result is proved by explicit calculation.

Theorem 10 For every $a \geq 2$ there is a constant $r_{0}(a)>4 a$ such that for all $2<r<r_{0}(a)$ the set $\left\{I_{1}=I_{4}=0\right\}$ contains an open cone of two dimensional tori which possess one hyperbolic normal degree of freedom. This cone is parameterised by $I_{2}>0$ and $I_{3} \in\left(I_{3}^{-}\left(I_{2}\right), I_{3}^{+}\left(I_{2}\right)\right)$. Each such torus corresponds to the line $L_{1}=L_{1}^{-}$in a reduced phase space whose phase portrait resembles that shown in Figure $7 \mathrm{~b}$; its stable and unstable manifolds are connected by a homoclinic orbit.

When both $I_{1}=I_{4}$ and $I_{2}=I_{3}$ the reduced phase space $P\left(L_{2}, L_{3}, L_{4}, a\right)$ has conical singularities at both poles, at which it intersects the $\left\{I_{1}=I_{4}=0\right\}$ and $\left\{I_{2}=I_{3}=0\right\}$ coordinate subspaces in stable two dimensional tori. There is always an elliptic critical point for $n$ even and a hyperbolic critical point for $n$ odd; the phase portrait therefore resembles that shown in Figure 7c.

Proposition 11 Each of the basic periodic orbits is stable.

Proof: Consider, for example, the $z_{2}$ basic periodic orbit. This orbit lies on the conical pole of a singular leaf, where $I_{1}=I_{3}=I_{4}=0$; the other pole is regular and corresponds to $I_{2}=0$. A direct calculation shows that the vector 
field (51), (52) vanishes at the conical pole only when

$$
r=\frac{4 a\left(3 a^{2}+3 a+1\right)\left(a^{3}+3 a^{2}+4 a+1\right)}{(a+1)^{5}}
$$

and for this value of $r$ it vanishes for each $\psi_{1} \in[0, \pi)$. The phase portrait therefore resembles that shown in Figure $5 \mathrm{a}$ or $5 \mathrm{~b}$ (with $L_{1}^{-}$and $L_{1}^{+}$interchanged) except that the vector field vanishes identically on $L_{1}=L_{1}^{-}$.

A similar examination of the other basic periodic orbits shows that the vector field never vanishes at the conical surface of the corresponding reduced phase spaces.

\section{Acknowledgement}

The first author would like to thank the Institut des Hautes Etudes Scientifique for its warm hospitality during the spring of 1996, where part of this research was performed.

\section{References}

[1] A. I Dyachenko and V. E. Zakharov, "Is free-surface hydrodynamics an integrable system?", Phys. Lett. A 190, 144-148 (1994).

[2] W. Craig and P. Worfolk, "An integrable normal form for water waves in infinite depth", Physica D 84, 513-531 (1995).

[3] I. Tadjbakhsh and J. B. Keller, "Standing surface waves of finite amplitude", J. Fluid Mech. 8, 442-451 (1960).

[4] S. A. Thorpe, "On standing internal gravity waves of finite amplitude", J. Fluid Mech. 32, 489-528 (1968).

[5] P. G. Drazin, "Kelvin-helmholtz instability of finite amplitude", J. Fluid Mech. 42, 321-335 (1970).

[6] J. W. Miles, "Weakly nonlinear waves in a stratified fluid: a variational formulation", J. Fluid Mech. 172, 499-512 (1986).

[7] F. Dias and T. J. Bridges, "Geometric aspects of spatially periodic interfacial waves", Stud. Appl. Math. 93, 93-132 (1994).

[8] T. B. Benjamin and T. J. Bridges, "Reappraisal of the Kelvin-Helmholtz problem. Part 1: Hamiltonian structure", J. Fluid Mech. 333, 301-325 (1997). 
[9] V. E. Zakharov, "Stability of periodic waves of finite amplitude on the surface of a deep fluid", Zh. Prikl. Mekh. Tekh. Fiz. 9, 86-94 (1968). (English translation J. Appl. Mech. Tech. Phys. 2, 190-194.)

[10] W. Craig, "Birkhoff normal forms for water waves", Contemp. Math. 200, 57-73 (1996).

[11] W. Craig and C. Sulem, "Numerical simulation of gravity waves", J. Comp. Phys. 108, 73-83 (1993).

[12] W. Craig and M. D. Groves, "Hamiltonian long-wave approximations to the water-wave problem", Wave Motion 19, 367-389 (1994). 